\title{
High-modulation-depth effects in photorefractive wave mixing: influence on pattern formation and physical foundations
}

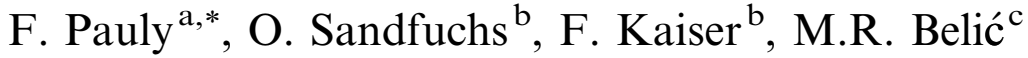 \\ ${ }^{a}$ Institut für Theoretische Festkörperphysik, Universität Karlsruhe, 76128 Karlsruhe, Germany \\ ${ }^{\mathrm{b}}$ Institute of Applied Physics - Nonlinear Dynamics, Darmstadt University of Technology, Hochschulstraße 4a, \\ 64289 Darmstadt, Germany \\ ${ }^{\mathrm{c}}$ Institute of Physics, P.O. Box 57, 11001 Belgrade, Yugoslavia
}

\begin{abstract}
Spatio-temporal pattern formation in contra-directional two-wave mixing in photorefractive crystals is investigated both numerically and analytically, taking high-modulation-depth effects into account. We use a modified two-wavemixing model that includes a correction function $f(m)$, which is introduced as a phenomenological modification. Transverse instabilities arise only if the modulation depth at one side of the crystal is high (near unity). Therefore, highmodulation-depth effects are both relevant and important. In the near field, transverse running waves appear above the instability threshold. The gain of the signal beam then exhibits an unexpected saturation. Furthermore, the physical justifications of the correction function $f(m)$ are investigated by exactly integrating the nonlinear Kukhtarev equations. We do not neglect the phases of the counter-propagating beams. For static gratings we obtain real and superlinear correction functions, whereas for running gratings they change to complex correction functions.
\end{abstract}

PACS: 42.65.-k; 42.65.Hw; 42.65.Sf

Keywords: Nonlinear optics; Photorefractive effect; Two-wave mixing; Transverse instabilities; Spatio-temporal dynamics; Highmodulation-depth effects

\section{Introduction}

The response of a photorefractive crystal to a light distribution is described by the band transport model, as developed by Kukhtarev et al. [1]. According to the Kukhtarev equations (KE), an interference pattern

\footnotetext{
${ }^{*}$ Corresponding author. Tel.: +49-721-608-6031; Fax: +49-721-608-7040.

E-mail address: Fabian.Pauly@tfp.uni-karlsruhe.de (F. Pauly).
} 
in the crystal builds up a space charge field that induces a refractive index modulation due to the Pockels effect. To explain two-wave-mixing (TWM) experiments the Maxwell equations are coupled to the KE via the Pockels effect, thereby including the change in the refractive index, which is caused by the interference of the coherent light beams [2]. A treatable system of differential equations is obtained by the use of several simplifying assumptions. One important assumption is the low contrast (modulation depth $m$ ) of the interference pattern [2]. Because of this, nonlinearities in the KE are ignored in the standard TWM equations. To extend the validity of TWM equations to the regime of high modulation depths, the introduction of modulation-depth-dependent-correction functions $f(m)$ has been suggested by Refregier et al. [3]. Up to now, a number of such functions $f(m)$ have been proposed, based either on the analysis of the KE or motivated by experimental findings [4-9]. Examples include $f(m)=\left(1-\mathrm{e}^{-a m}\right) / a[3], f(m)=m /(1+b m)$ [4], while $f(m)=m$ represents the function obtained in the small- $m$ approximation [2].

In recent years transverse instabilities in contra-directional TWM attracted considerable interest. Since the initial observation of hexagonal patterns in the optical far field of a feedback configuration [10-12], many additional structures, such as stripes or squares, have been identified [13,14]. With the help of linear stability analyses a better understanding of the origin of these structures is achieved [15-17]. In [17] the characteristics of pattern formation above the instability threshold were additionally explored in a numerical simulation. However, high-modulation-depth effects have not been taken account of in these contributions. They are the subject of the present publication.

The paper is divided into two main parts. In the first part spatio-temporal pattern formation in contra-directional TWM is investigated both numerically and analytically, taking a high modulation depth into account. The correction function $f(m)=m /(1+b m)$, as proposed by Kwak et al. [4], is used for this purpose. The parameter $b$ introduces in a phenomenological way the nonlinear response of the photorefrective crystal into the TWM. This response is sublinear (superlinear) for positive (negative) values of the saturation $b$, while the usual linear response is obtained for $b=0$. At variance with the original proposal by Kwak et al. [4], who suggests saturations $b>0$, we will substantiate (in the second part of this publication) that such a sublinear response is not appropriate for describing static gratings. Therefore we will consider the whole range of saturations (positive, zero and negative) in the first part of our paper. Numerical simulations are performed using a relaxation method [18]. Analytical results are obtained by a non-autonomous stability analysis, considering variations of the modulation depth along the direction of beam propagation. The high-modulation-depth effects are relevant, because we demonstrate, that in contra-directional TWM the modulation depth at one face of the crystal is near unity at the instability threshold. We discuss the properties of the observed patterns and other significant TWM quantities.

In the second part, the foundations of high-modulation-depth corrections, based on the underlying KE, are discussed. We investigate numerical solutions of the band transport model with a new spectral integration scheme. The space charge field is computed for stationary intensity patterns, with and without applied electric fields, as well as for moving patterns. We show that, for the correct consideration of the phases of the interacting beams, superlinear real correction functions need to be used for the static case, and complex valued correction functions for moving interference fringes.

\section{Pattern formation including high-modulation-depth effects}

Contra-directional TWM in one transverse dimension is described by the equations given in [17]

$$
\begin{aligned}
& \partial_{z} A_{1}+\mathrm{i} F \partial_{x}^{2} A_{1}+\alpha A_{1}=-Q A_{2}, \\
& -\partial_{z} A_{2}+\mathrm{i} F \partial_{x}^{2} A_{2}+\alpha A_{2}=Q^{*} A_{1},
\end{aligned}
$$




$$
\tau \partial_{t} Q+\eta Q=\frac{\Gamma}{2} f(m) \mathrm{e}^{\mathrm{i}\left(\psi_{1}-\psi_{2}\right)}
$$

In Eq. (3) the modulation depth $m$ is replaced by the generalized function $f(m)$.

The propagation of two counter-propagating beams in the photorefractive medium (Fig. 1) is expressed by Eqs. (1) and (2). $A_{i}(x, z, t)$ are the (complex) slowly varying envelopes of the light fields. The negative sign in Eq. (2) in front of the derivative with respect to $z$ - the propagation direction - is due to the opposite direction of beam two. The term $F=L /\left(2 k_{0} w_{0}^{2}\right)$ represents the diffraction in the transverse dimension $x$ and is proportional to the inverse Fresnel number. $L$ denotes the longitudinal length of the crystal and $k_{0}$ the wave vector of each beam in the crystal along the longitudinal direction. The transverse coordinate has been scaled to the beam waist $w_{0}$. The parameter $\alpha$ describes the linear absorption. It is neglected in this paper. Both beams couple through their interaction in the photorefractive crystal, which is described by the variable

$$
Q=-\mathrm{i} L k_{0} n_{0}^{2} r_{\mathrm{eff}} E_{1}^{\mathrm{sc}} / 4 .
$$

Here $n_{0}$ is the ordinary refractive index of the crystal, $r_{\text {eff }}$ the effective electro-optical coefficient and $E_{1}^{\text {sc }}$ stands for the first Fourier component of the space charge field.

Eqs. (1) and (2) follow from the Maxwell equations, assuming light fields $E_{i}(x, z, t)=A_{i}(x, z, t)$ $\exp \left(-\mathrm{i}\left(\vec{k}_{i} \vec{r}-\omega t\right)\right)$ outside and inside the crystal [2]. For light fields of this kind a beam coupling is mainly due to a periodic refractive-index variation with the grating wave vector $\vec{K}_{\mathrm{g}}=\vec{k}_{2}-\vec{k}_{1}$, because modulations at a wave number of the grating wave vector are the only resonant components in the longitudinal direction. Since this refractive-index variation is linearly connected (via the Pockels effect) to the space charge field, the first Fourier component of the space charge field is the dominant quantity for beam coupling.

Eq. (3) determines the temporal evolution of the first Fourier component of the space charge field $E_{1}^{\text {sc }}$ $\left(Q \propto E_{1}^{\text {sc }}\right.$, according to Eq. (4)). This equation is derived from the KE (see Section 3), which describe the response of the crystal to an intensity pattern. It is a crucial point, that a small fringe contrast of the interference pattern, characterized by a modulation depth $m \ll 1$ with

$$
m:=\frac{2\left|A_{1}\right|\left|A_{2}\right|}{\left|A_{1}\right|^{2}+\left|A_{2}\right|^{2}},
$$

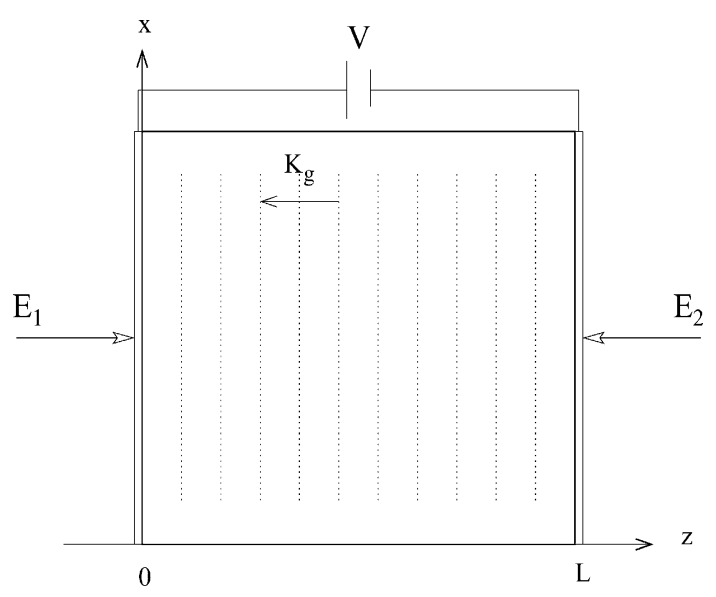

Fig. 1. Experimental setup: Two beams $E_{1}$ and $E_{2}$ are propagating into opposite directions in a photorefractive crystal. The externally applied electric field $E_{0}=V / L$ is adjusted by an external voltage $V$. 
is assumed in this derivation. Therefore, the KE are linearized, and all higher harmonics, except the first Fourier component in the grating wave vector $\vec{K}_{\mathrm{g}}$, are neglected. Furthermore, spatial derivatives of the Fourier components are ignored, assuming that the crystal's internal properties depend only on $z$. If these steps are carried out, the modulation-depth-dependent function $f(m)$ becomes equal to the modulation depth $(f(m)=m)$. To take into account the nonlinearities of the crystal response at high modulation depth $(m \approx 1)$, Refregier et al. [3] introduced a phenomenological correction $f(m)$. Such a modification is incorporated into our TWM model as well. As already tried in [3], these correction functions should be determinable from the KE.

The variables $\psi_{i}(i=1,2)$ in Eq. (3) are the phases of the envelopes $A_{i}=\left|A_{i}\right| \exp \left(\mathrm{i} \psi_{i}\right)$ and $\tau$ stands for the characteristic photorefractive response time. The coupling parameter $\Gamma$ between the beams and the induced space charge field $\left(E_{1}^{\text {sc }} \propto Q\right)$ is given by

$$
\Gamma=\Gamma_{0}\left(1+\frac{E_{\mathrm{q}}}{E_{\mathrm{d}}}\right) \frac{E_{\mathrm{d}}+\mathrm{i} E_{0}}{E_{\mathrm{q}}+E_{\mu}+\mathrm{i} E_{0}}
$$

and the relaxation constant by

$$
\eta=\frac{\left(E_{\mathrm{d}}+E_{\mathrm{q}}+\mathrm{i} E_{0}\right)}{\left(E_{\mathrm{d}}+E_{\mu}+\mathrm{i} E_{0}\right)} .
$$

$\eta$ and $\Gamma$ depend on an externally applied electric field $E_{0}$ (see Fig. 1). By this means the response of the crystal to light can be changed. $E_{\mathrm{d}}, E_{\mu}$ and $E_{\mathrm{q}}$ are the characteristic fields of the crystal: the diffusion field, the drift field and the limiting space charge field, respectively. These fields are chosen to be $E_{\mathrm{d}}=1 \mathrm{kV} / \mathrm{cm}$, $E_{\mu}=100 E_{\mathrm{d}}$ and $E_{\mathrm{q}}=5 E_{\mathrm{d}}$ in the TWM simulations, describing a $\mathrm{BaTiO}_{3}$-crystal [19]. $\Gamma_{0}$ is set to 2 and $F$ to 0.025 .

To summarize, the TWM model given by Eqs. (1)-(3) is consistently considering only first Fourier components in the grating wave vector $\vec{K}_{\mathrm{g}}$ for small intensity contrast, if the correction function is chosen to be $f(m)=m$. As the first Fourier component of the space charge field is the only resonant coupling term for the two beams, their interaction due to higher harmonic gratings in the refractive index is assumed to be low. This assumption seems plausible, since the grating wave length of an interference pattern (for visible light with $\lambda=500 \mathrm{~nm}$ ) is in the order of $1 \mu \mathrm{m}$. For a crystal of length $1 \mathrm{~cm}$, there are about four orders of magnitude between the two length scales. Consequently, the non-resonant components should quickly be averaged out in the propagation equations for the slowly varying envelopes $A_{1}$ and $A_{2}$. This justifies the use of the first two TWM Eqs. (1) and (2) even in the presence of higher-harmonic-refractive-index gratings. With the help of the modification $f(m)$, instead of $m$, one hopes to extend the validity of Eq. (3) in a simple manner, to account for nonlinear modulation-depth-dependent effects of the resonant space-charge-field component $E_{1}^{\text {sc }}$. It should be remembered, that by such a procedure the higher harmonic components, present at high modulation depths, are not taken into account with respect to their possible coupling to the beams.

The boundary conditions in contra-directional TWM consist in the fixed beam profiles at $z=0$ for beam one and $z=L$ for beam two. As only relative intensities play a role in our model, we define the beam ratio $r_{10,2 L}$ as

$$
r_{10,2 L}:=\frac{I_{1}(x=0, z=0)}{I_{2}(x=0, z=L)} .
$$

For TWM simulations we use Gaussian beams incident upon opposite faces of the crystal ${ }^{1}$

\footnotetext{
${ }^{1}$ Scaling the transverse direction to the beam waist $w_{0}$, as mentioned in the discussion of Eqs. (1) and (2), we do not have to assume an absolute value for this quantity.
} 


$$
\begin{aligned}
& A_{1}(x, z=0)=\left|A_{1}(x=0, z=0)\right| \mathrm{e}^{-x^{2} / w_{0}^{2}}, \\
& A_{2}(x, z=L)=\left|A_{2}(x=0, z=L)\right| \mathrm{e}^{-x^{2} / w_{0}^{2}},
\end{aligned}
$$

and we may set $\left|A_{1}(x=0, z=0)\right|=1$ and $\left|A_{2}(x=0, z=L)\right|=\left(r_{10,2 L}\right)^{-1 / 2}$.

\subsection{Linear stability of transverse modes}

To obtain information on the instability threshold, we perform a linear stability analysis. Plane-wave solutions of Eqs. (1)-(3) are discussed in contrast to the Gaussian beams, which we use in our TWM simulations (see Eqs. (9) and (10)). We use the ansatz

$$
\left(\begin{array}{l}
A_{1}(x, z, t) \\
A_{2}(x, z, t) \\
Q(x, z, t)
\end{array}\right)=\left(\begin{array}{l}
A_{1}^{0}(z)\left(1+\epsilon a_{1}(x, z, t)\right) \\
A_{2}^{0}(z)\left(1+\epsilon a_{2}(x, z, t)\right) \\
Q^{0}(z)(1+\epsilon q(x, z, t))
\end{array}\right),
$$

where $a_{1}, a_{2}$ and $q$ are small perturbations to the plane-wave solutions, which are decomposed into the transverse Fourier $(x \rightarrow K)$ and temporal Laplace $(t \rightarrow \lambda)$ components. The procedure is similar to the one in [17], where the simpler case $f(m)=m$ has been treated in detail. Variables marked with the superscript 0 indicate steady-state-plane-wave solutions and therefore carry only a $z$-dependence. After the ansatz solutions are substituted in the starting equations, the equation for $q$ turns out to be purely algebraic, and hence $q$ can be eliminated. The four linearized equations can now be written as

$$
\mathscr{T} \partial_{z} \vec{s}_{\text {per }}=\widetilde{A}\left[F K^{2}, \lambda, E_{0}, m^{0}, f\left(m^{0}\right), f^{\prime}\left(m^{0}\right)\right] \mathscr{T} \vec{s}_{\text {per }},
$$

where $\vec{s}_{\text {per }}=\left(a_{1+}, a_{1-}, a_{2+}, a_{2-}\right)^{\mathrm{T}}$ contains the Fourier-Laplace coefficients of the perturbations and $\mathscr{T}$ is a transformation into a real basis. The prime in $f^{\prime}\left(m^{0}\right)$ denotes a differentiation of the correction function with respect to the modulation depth. The stability matrix is given by

$$
\widetilde{A}=\left(\begin{array}{cccc}
\left(m^{0}\right)^{2} D_{3}^{\mathrm{r}}+\left(1-\left(m^{0}\right)^{2}\right)\left(D_{1}^{\mathrm{r}}+D_{2}^{\mathrm{r}}\right) & -F K^{2} & 0 & -s \sqrt{1-\left(m^{0}\right)^{2}} D_{1}^{\mathrm{i}} \\
F K^{2}+\left(m^{0}\right)^{2} D_{3}^{\mathrm{i}}+\left(1-\left(m^{0}\right)^{2}\right)\left(D_{1}^{\mathrm{i}}+D_{2}^{\mathrm{i}}\right) & 0 & 0 & s \sqrt{1-\left(m^{0}\right)^{2}} D_{1}^{\mathrm{r}} \\
s \sqrt{1-\left(m^{0}\right)^{2}}\left(D_{1}^{\mathrm{r}}+D_{2}^{\mathrm{r}}\right) & 0 & 0 & -F K^{2}-D_{1}^{\mathrm{i}} \\
s \sqrt{1-\left(m^{0}\right)^{2}}\left(D_{1}^{\mathrm{i}}+D_{2}^{\mathrm{i}}\right) & 0 & F K^{2} & D_{1}^{\mathrm{r}}
\end{array}\right) .
$$

We use the abbreviations $D_{j}^{\mathrm{r}}=\operatorname{Re}\left(D_{j}\right), D_{j}^{\mathrm{i}}=\operatorname{Im}\left(D_{j}\right)$, as well as $D_{1}=\lambda \Gamma_{\mathrm{e}} \tau_{\mathrm{e}}\left[f\left(m^{0}\right) / m^{0}\right] /\left(1+\lambda \tau_{\mathrm{e}}\right)$, $D_{2}=\Gamma_{\mathrm{e}}\left[\left(f\left(m^{0}\right) / m^{0}\right)-f^{\prime}\left(m^{0}\right)\right] /\left(1+\lambda \tau_{\mathrm{e}}\right)$ and $D_{3}=\Gamma_{\mathrm{e}}\left[f\left(m^{0}\right) / m^{0}\right]^{2}$. In this short-hand notation the complex quantity $\lambda=\sigma+\mathrm{i} \Omega$ must not be conjugated when writing $D_{j}^{\mathrm{r}}$ and $D_{j}^{\mathrm{i}}$. The effective time constant $\tau_{\mathrm{e}}=\tau / \eta$ and coupling constant $\Gamma_{\mathrm{e}}=\Gamma / \eta$ have been introduced.

Generally the stability matrix $\widetilde{A}$ is $z$-dependent through the modulation depth $m^{0}(z)$ of the plane-wave solutions ${ }^{3}$. The term $D_{2}$ does not exist in the linear model, because the difference $f\left(m^{0}\right) / m^{0}-f^{\prime}\left(m^{0}\right)$ vanishes for $f\left(m^{0}\right)=m^{0}$. It is important for the instability threshold, which of the two beams is more intense, as indicated by $s=\operatorname{sign}\left(I_{2}^{0}-I_{1}^{0}\right)$.

\footnotetext{
${ }^{2}$ These expressions already account for complex correction functions, which are introduced in Section 3.2.

${ }^{3}$ If the absorption $\alpha$ is included, the effect on the instability threshold (as stated in [17]) is given in an indirect manner, via the influence on $m^{0}(z)$. The absorption would not cause the appearance of additional terms in this more general case of an arbitrary $f(m)$.
} 
To discuss the onset of transverse instabilities, it is necessary to integrate Eq. (12). If the modulation depth $m^{0}(z)$ is known at $z=0$, one can determine the resolvent of the stability problem. Owing to the counter-propagating beams, the poles of a scattering matrix determine the instability threshold.

\subsection{Determination of the z-dependent modulation depth}

In this section we want to determine the $z$-dependence of the plane-wave modulation depth $m^{0}$ for given external field strength $E_{0}$, intensity ratio $r_{10,2 L}$ and parameters of $f\left(\mathrm{~m}^{0}\right)$, because the stability of stationary plane waves $\left(\partial_{t} Q=0, \partial_{x}^{2} A_{j}=0\right)$ is to be discussed (see Sections 2.3 and 2.4). The TWM equations then simplify significantly, since the differential equations for phases and intensities decouple. The modulation depth obeys the following differential equation:

$$
\partial_{z} m^{0}=-\gamma f\left(m^{0}\right)\left[1-\left(m^{0}\right)^{2}\right] .
$$

Here $\gamma=\operatorname{Re}\left(\Gamma_{\mathrm{e}}\right)$ is the real part of the effective coupling constant $\Gamma_{\mathrm{e}}$. Its sign depends on the orientation of the crystal's $c$-axis. Assuming that the beam, incident upon the crystal at $z=0$ (pump beam), is decreasing in energy $(\gamma \geqslant 0)$, it follows that for $m^{0}(z=0)=1$ the modulation depth is constant $\left(m^{0}(z) \equiv 1\right)$ within the whole crystal. In all other cases $\left(m^{0}(z=0) \neq 1\right)$ the modulation depth $m^{0}$ decreases monotonically with $z$. To obtain the maximum value of the modulation depth $\left(m^{0}=1\right)$, the intensity ratio has to be chosen as

$$
r_{10,2 L}=\exp [\gamma f(1) L] .
$$

We apply the method given by Belić et al. [7] to determine the modulation depth for the general case when $r_{10,2 L}$, the applied field strength $E_{0}$ and the parameters of the correction function $f(m)$ can be independently varied. The aim is to compute $m^{0}(z=0)$ and then to integrate the modulation depth over the length of the crystal, according to Eq. (14). Throughout the first part of the paper we will exclusively use the function $f(m)=m /(1+b m)$ [4]. We will discuss the whole range of crystal behaviour, namely sublinear $(2 \geqslant b>0)$, linear $(b=0)$ and superlinear $(-0.5 \leqslant b<0)$. The strength and sign of the saturation $b$ depends on the parameters of the photorefractive crystal and on the interference pattern inside the crystal itself ${ }^{4}$. It describes the crystal's characteristic behaviour. With this specific choice of $f(m)$ we need to solve the following equations:

$$
\begin{aligned}
& (1-b)\left(\log \left(\cosh \left(\frac{H_{L}}{2}\right)\right)-\log \left(\cosh \left(\frac{H_{0}}{2}\right)\right)\right) \\
& +(1+b)\left(\log \left(\sinh \left(\frac{H_{L}}{2}\right)\right)-\log \left(\sinh \left(\frac{H_{0}}{2}\right)\right)\right)-\gamma L=0, \\
& H_{L}+H_{0}+\log \left(\sinh \left(\frac{H_{L}}{2}\right)\right)-\log \left(\sinh \left(\frac{H_{0}}{2}\right)\right)-\log \left(r_{10,2 L}\right)=0,
\end{aligned}
$$

for $H_{0}=H(z=0)$ and $H_{L}=H(z=L) . H$ is connected to the modulation depth by $m^{0}(z)=\operatorname{sech}[H(z)]$.

An example of the modulation-depth dependence on $z$ is shown in Fig. 2. For the saturations (a) $b=0$ and (b) $b=1.5$, the modulation depth $m^{0}(z=0)$ can be increased by the use of the external field $E_{0}{ }^{5}$. The modulation depth at $z=0$ is decreasing for higher saturation $b$, as might be expected, due to an effectively reduced beam coupling. [See the factor $(1+b m)^{-1}$ in Eq. (3), compared to the standard TWM equations $(f(m)=m)$.] This decreasing at $z=0$ is also observed for higher intensity ratios $r_{10,2 L}$ (not shown here).

\footnotetext{
${ }^{4}$ This latter statement will be demonstrated in the second part of this publication, where a crossover from superlinear to sublinear response is observed when static interference patterns start to move.

${ }^{5}$ A negative electric field is pointing in the negative $z$-direction.
} 

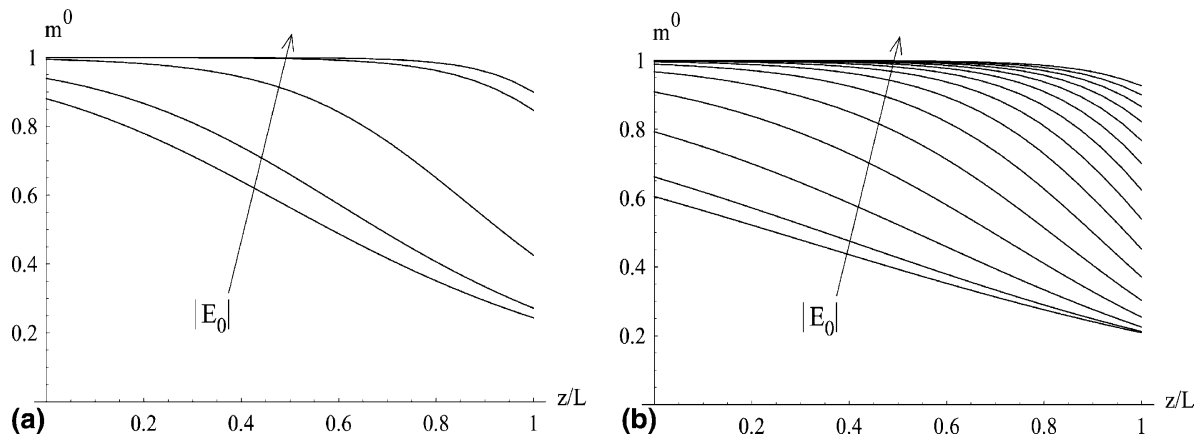

Fig. 2. Longitudinal modulation-depth dependence $m^{0}(z)$ for plane waves at different saturations and applied external field strengths. (a) $b=0, r_{10,2 L}=100, E_{0}=0$ to $-4 E_{\mathrm{d}}$, (b) $b=1.5, r_{10,2 L}=100, E_{0}=0$ to $-14 E_{\mathrm{d}} . E_{0} / E_{\mathrm{d}}$ has been decreased in steps of 1 in the direction of the arrow.

Concerning the longitudinal behaviour, the modulation depth $m^{0}$ is decaying with $z$, according to Eq. (14), and stays close to unity in a certain region around $z=0$, if its starting value is high. How far this area of high modulation depth is extended, depends on $\gamma$. For high beam ratios $r_{10,2 L}$ the modulation depth $m^{0}$ decreases quickly, because a high intensity coupling $\gamma$ is necessary to reach a modulation depth $m^{0}(z=0)$ near unity. This will be shown in Section 2.4, together with the observation, that at the instability threshold the modulation depth at $z=0$ is nearly at its maximum value $\left(m^{0}(z=0) \approx 1\right)$.

\subsection{Threshold characteristics for modulation depth one}

The linear stability of plane-wave solutions for a modulation depth equal to one $\left(m^{0}=1\right)$ is the subject of the present section. In this special case the beam ratio $r_{10,2 L}$ is fixed for a given saturation $b$ and an electric field $E_{0}$ (see Eq. (15)). An analytical expression for the threshold condition can be derived, owing to the constant modulation depth [17]. In our case, $\Gamma_{\mathrm{e}}$ is modified with a factor of $f(1)=(1+b)^{-1}$.

In this section we will only discuss the critical coupling $\Gamma_{\mathrm{e}}^{\mathrm{c}}$ and scaled critical coupling $\Gamma_{\mathrm{e}}^{\mathrm{c}} /(1+b)$ (Fig. 3(a) and (b)), because other critical parameters and pattern characteristics (as for example the critical electric field) closely resemble the threshold curves obtained for $r_{10,2 L}=25$ in Section 2.4.

The magnitude of the critical coupling $\Gamma_{\mathrm{e}}^{\mathrm{c}}$ increases for higher saturations $b$. This is achieved via a higher magnitude of $E_{0}$. We emphasize, that the real part of the scaled coupling, $\gamma^{\mathrm{c}} /(1+b)$, is only slightly
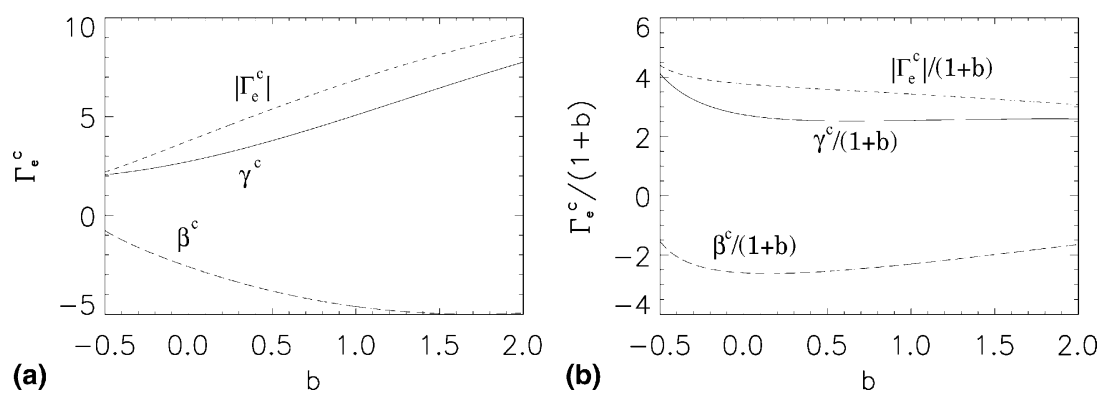

Fig. 3. (a) Critical coupling $\Gamma_{\mathrm{e}}^{\mathrm{c}}$ and (b) scaled critical coupling $\Gamma_{\mathrm{e}}^{\mathrm{c}} /(1+b)$ as a function of the saturation $b . \gamma$ and $\beta$ are the real and imaginary parts of the effective coupling constant $\Gamma_{\mathrm{e}}$, respectively. 
changing for $b>0$ (Fig. 3(b)). As $\gamma^{\mathrm{c}} /(1+b)$ is the coupling between the intensities of the counter-propagating beams, transverse instabilities arise in this sublinear region nearly for the same intensity coupling at the modulation depth of one. Another aspect of this approximately constant $\gamma^{\mathrm{c}} /(1+b)$ is the fixed beam ratio $r_{10,2 L}$ in the sublinear region, according to Eq. (15). This signifies that, even for "different sublinear photorefractive crystals", the instability is occurring at the same beam ratio. However, the changes of $\gamma^{\mathrm{c}} /(1+b)$ and $\beta^{\mathrm{c}} /(1+b)$ in the region of superlinear response indicate, that it is a strong simplification to characterize the pattern formation only by the intensity coupling. The description of the TWM system demands the complete threshold equation.

\subsection{Threshold and pattern characteristics for z-dependent modulation depth}

In the following we will discuss results for $z$-dependent modulation depth. The threshold condition is computed by integrating the non-autonomous problem given in Eq. (12), using a Runge-Kutta integration scheme of fourth order. The modulation depth at $z=0$ is determined as described in Section 2.2. Predictions of the non-autonomous stability analysis for plane waves will be compared with the numerical results obtained by the simulation of the TWM process. In our TWM simulations we integate the full Eqs. (1)-(3) for incident Gaussian beams (see Eqs. (9) and (10)). The values of the modulation depth at $z=0$ and $z=L$ for different saturations and intensity ratios are displayed in Fig. 4 and the longitudinal behaviour of the modulation depth $m(x=0, z)$, as obtained for Gaussian beams, is represented in Fig. 5. (In these diagrams the modulation depth is not indicated by the superscript 0 , because the modulation-depth dependence of Gaussian beams under diffraction is not the same as that of the plane waves (see Eq. (14).)

The high modulation depth in the region of $z=0$ is recognized as a characteristic of the instability threshold, being nearly unity for all beam ratios and saturations $b$ (Figs. 4 and 5). The case $r_{10,2 L}=25$ is quite close to the constant modulation depth $m=1$ (see Section 2.3), because for this beam ratio the modulation depth is hardly departing from unity within the crystal. However, the modulation depth is generally not constant over the whole range of the crystal, but decreases monotonically with increasing $z$. At the instability threshold the longitudinal behaviour of the modulation depth is nearly the same for different saturations $b$, which is achieved by an adjustment of $E_{0}$ (Figs. 4 and 5(a)). Note that this is only

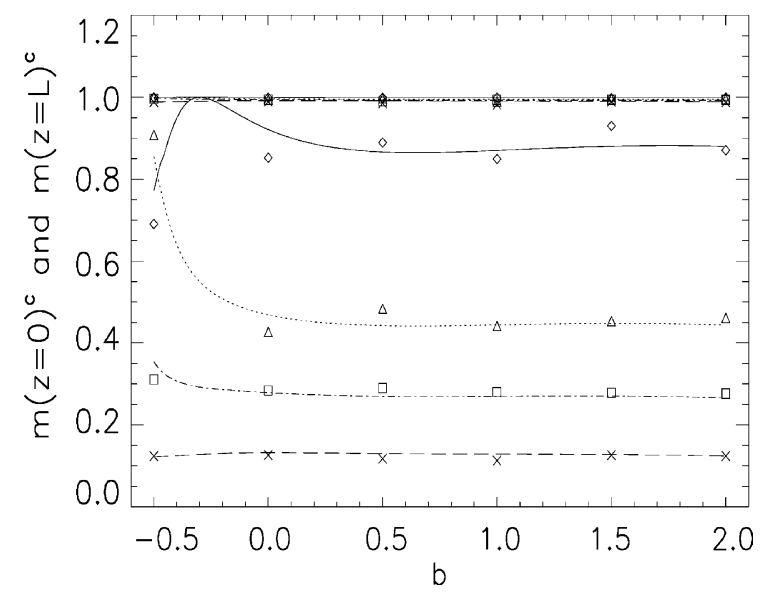

Fig. 4. Comparison between the critical modulation depth $m^{0}(z)$ of plane waves determined using a non-autonomous stability analysis (lines) and the modulation depth $m(x=0, z)$ of the Gaussian beams (symbols) slightly below the transverse instability threshold. Shown are the critical modulation depths for different intensity ratios $r_{10,2 L}$ as a function of $b$ at $z=0$ and $z=L$. The modulation depths $m(z=0)^{\mathrm{c}}$ are all close to one, but the modulation depths $m(z=L)^{\mathrm{c}}$ can be distinguished by the different symbols and line styles. $(\diamond$ and -$) r_{10,2 L}=25,(\triangle$ and $\cdots) r_{10,2 L}=100,(\square$ and ---$) r_{10,2 L}=250,(\times$ and $-\cdot-\cdot) r_{10,2 L}=1000$. 

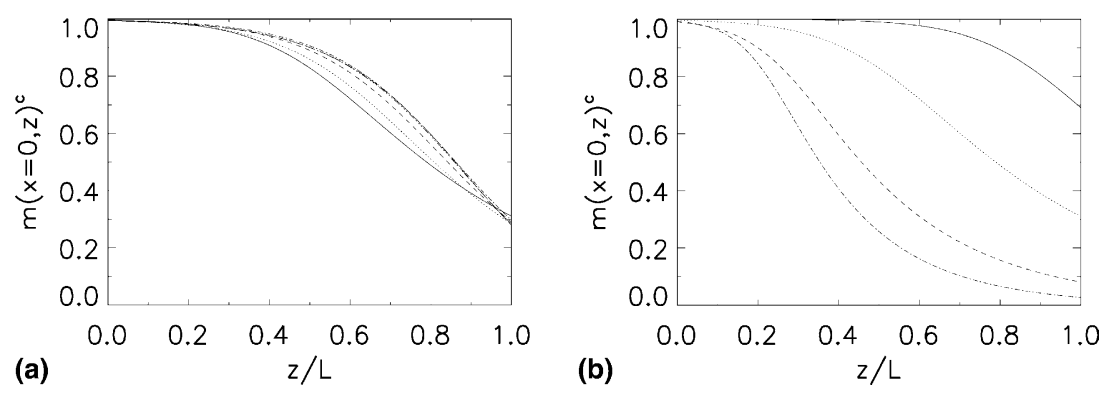

Fig. 5. The longitudinal behaviour of the critical modulation depth $m(x=0, z)^{\mathrm{c}}$ for different saturations $b$ and intensity ratios $r_{10,2 L}$ as obtained from numerical simulations with Gaussian beams. (a) $(-) b=-0.5,(\cdots) b=0,(--) b=0.5,(-\cdots) b=1,(-\cdots \cdots-) b=1.5$, $(---) b=2$ and $r_{10,2 L}=250$, respectively, (b) $(-) r_{10,2 L}=25,(\cdots) r_{10,2 L}=250,(--) r_{10,2 L}=2500,(-\cdots) r_{10,2 L}=2.5 \times 10^{4}$ and $b=-0.5$, respectively.

true as long as $\Delta=I_{1}-I_{2}$ is positive. Below $b=-0.3$ for the beam ratio $r_{10,2 L}=25$ the "pump beam" $I_{1}$ is weaker than the "signal beam" $I_{2}$ and a strong variation of $m(z=L)$ is observable in the region of the crossover. (The same crossover phenomenon can already be anticipated for $r_{10,2 L}=100$ from Fig. 4 . As a simple rule of thumb - ignoring the finite critical field strength - Eq. (15) predicts $b \approx-0.4$ for $r_{10,2 L}=25$ and $b \approx-0.58$ for $r_{10,2 L}=100$ to be the points of modulation depth one.) For different beam ratios the curve shape of the modulation depth varies considerably, and it is decaying faster towards the crystal's end for high $r_{10,2 L}$ (Figs. 4 and 5(b)). Thus, for high intensity ratios there is a significant departure from $m(z) \equiv 1$.

In Fig. 6 the critical values of the field strength $E_{0}^{\mathrm{c}}$, together with the critical oscillation frequency $\Omega^{\mathrm{c}}$ and the mode parameter $\left(F K^{2}\right)^{\mathrm{c}}$ are plotted as functions of the saturation $b$ for different intensity ratios (see below Eq. (13)). Considering the condition $m(z=0)^{\mathrm{c}} \approx 1$ at the instability threshold, the behaviour of the critical variables can be understood as follows: Fig. 6 (a) shows, that $\left|E_{0}^{\mathrm{c}}\right|$ increases with $b$. This is similar to the autonomous case $(m=1)$, in which the coupling is effectively reduced with increasing $b$, and higher magnitudes of the electric field are necessary to reach the instability threshold. For higher beam ratios $r_{10,2 L}$, the magnitude of the applied field $\left|E_{0}^{\mathrm{c}}\right|$ increases as well, because the higher intensity difference $\Delta$ between the beams needs to be reduced by a higher coupling, in order to achieve high modulation depths at $z=0$.

The oscillation frequency decreases with decreasing saturation, when starting from a highly sublinear response (Fig. 6(b)) and indicates a lowering of the critical detuning of the side-band beams. This fact seems plausible, if one takes a closer look at the relaxation frequency $\eta / \tau$ in Eq. (3). This can, however, not explain the increase of $\Omega^{\mathrm{c}}$ for even lower values than $b \approx 0$, because $\eta / \tau$ is solely a function of the field strength. This function increases monotonically (in the magnitude of its real and imaginary part) with $E_{0}$ for
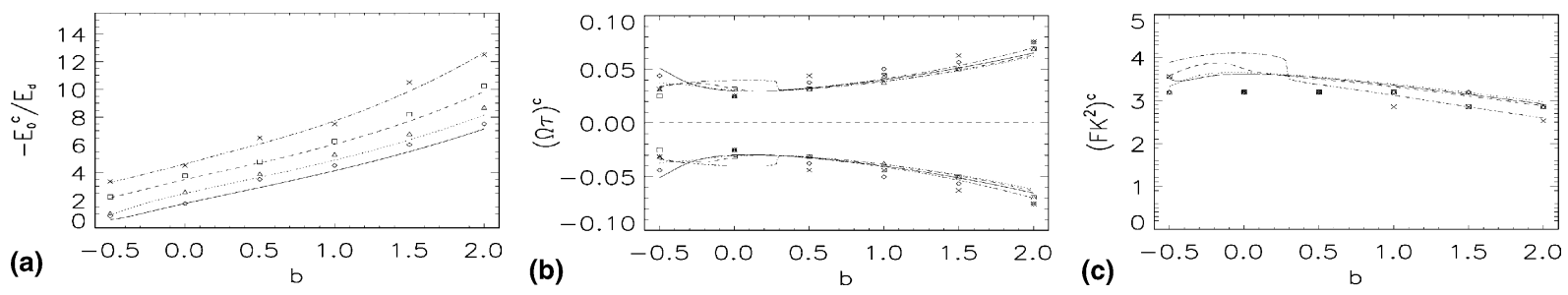

Fig. 6. Comparison between the predictions of the non-autonomous threshold condition for plane waves (lines) and the TWM simulation with Gaussian beams (symbols). (a) The critical electric field $E_{0}^{\mathrm{c}}$, (b) the critical oscillation frequency $\Omega^{\mathrm{c}}$ and (c) the critical mode parameter $\left(F K^{2}\right)^{\mathrm{c}}$ are shown as functions of the saturation $b$ for different intensity ratios $r_{10,2 L} .(\diamond$ and -$) r_{10,2 L}=25,(\triangle$ and $\cdots)$ $r_{10,2 L}=250$, $(\square$ and ---$) r_{10,2 L}=2500$, $(\times$ and $-\cdots) r_{10,2 L}=2.5 \times 10^{4}$. 
the parameters chosen in this paper. As $E_{0}^{\mathrm{c}}$ drops for decreasing $b$ for all the beam ratios studied here, this argument can only account for the lowerings in $\Omega^{\mathrm{c}}$. Analogously to the "modulation depth one", it needs to be stressed that the spontaneous response of the nonlinear TWM system demands the treatment of the complete threshold condition.

The transverse pattern size of the Gaussian beams corresponds qualitatively to the values calculated from the threshold condition (Fig. 6(c)), that predicts bigger transverse structures for higher $b$ in the sublinear response region. The values given here for the Gaussian beams are the transverse wave numbers of that mode in the far field of the signal beam with the temporally averaged maximum.

In Fig. (6) a pronounced jump in the critical oscillation frequency and transverse pattern can be observed for a beam ratio of $r_{10,2 L}=2.5 \times 10^{4}$, whereas the critical field $E_{0}^{\mathrm{c}}$ does still show a smooth behavior. The next smaller beam ratio $\left(r_{10,2 L}=2500\right)$ demonstrates a similar but more continuous behaviour. These results are therefore most likely not of a numerical nature, even if the TWM simulations deviate from the predictions of the stability analysis in this region. It seems as if a second transverse mode takes over at the threshold and also the simulations demonstrate a second mode with higher $K^{\mathrm{c}}$. But its amplitude is lower than the amplitude of the $K^{\mathrm{c}}$-mode plotted. This may be due to a the finite transverse extension of the Gaussian beams, compared to the planes waves used in the stability analysis. (An investigation of a dependence on the aspect ratio $\kappa$, see definition below, could be interesting in this regime.)

In the determination of the critical quantities using our TWM simulations, it is a general problem that, because of the small modulations observed at the instability threshold (arising from supercritial Hopf-bifurcations as shown in the next section), the instability boundary can only be approximated. Critical quantities, such as the oscillation frequency, are thus identified slightly above the threshold because a finite modulation needs to indicate, that a transverse instability is present. Furthermore, the discrepancies between the simulations and semi-analytics can be explained by the use of Gaussian beams in comparison to the stability analysis with plane waves. Only at very high aspect ratios $\kappa$, i.e., at high numbers of transverse modulations on the Gaussian beam profile, the results should be the same. The aspect ratio is defined as $\kappa=l K^{\mathrm{c}} / \pi$, where $l$ is the transverse width, on which the structure is imprinted. We set $l=w_{0}$, the beam waist of the Gaussian beam. In the simulations a $\kappa$ of approximately 4 was observed, while for plane waves we have $\kappa=\infty$. Under these considerations, the predictions of the stability analysis match the values obtained from the simulations very well.

\subsection{Temporal dynamics and pattern characteristics}

Below the instability threshold of the TWM simulations the system is in a temporally stationary state. The beams are counter-propagating and the signal beam is amplified by the pump beam. Above the threshold transverse running waves are observed in the near field at either side of the crystal (Fig. 7). From the excitation center in the middle of the beams, the waves are moving outwards along the Gaussian carrier wave. The structures behave temporally periodic and this behaviour is neither changed by different intensity ratios $r_{10,2 L}$ nor different saturations $b$. Compared to the situation shown in (a) and (b) for $b=1.5$, $r_{10,2 L}=250$ and $E_{0}=-7.0 E_{\mathrm{d}}$ only the external field strength was increased to $E_{0}=-8.5 E_{\mathrm{d}}$ in (c) and (d) ${ }^{6}$. Owing to the higher magnitude of the applied field, the amplitudes of the transverse modulations are strongly enhanced and the oscillation frequency increases. The stronger modulations signify a stronger energy transfer to side-band beams, which can be observed in the optical far field after a Fourier transformation of the complex envelopes. Two temporally changing peaks at the critical wave numbers $\pm K^{\mathrm{c}}$

\footnotetext{
${ }^{6}$ We have chosen a positive saturation as it will be shown that the sublinear response is the relevant for a moving grating, and thus for the discussion of TWM above the threshold, where a dynamical transverse instability has occured. In contrast a superlinear response is appropriate for static gratings or the discussion of critical quantities.
} 

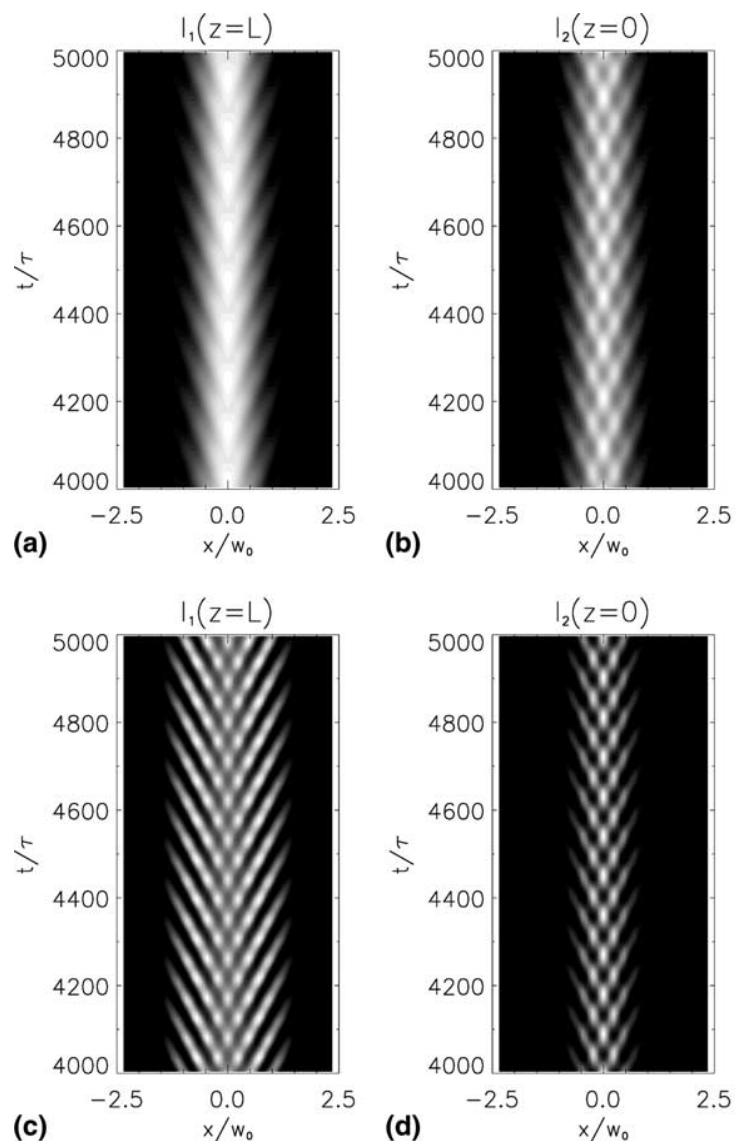

Fig. 7. Transverse running waves in the near field: $I_{1}(x, z=L)$ and $I_{2}(x, z=0)$ for $b=1.5, r_{10,2 L}=250$ and $(\mathrm{a}, \mathrm{b}) E_{0}=-7.0 E_{\mathrm{d}},(\mathrm{c}, \mathrm{d})$ $E_{0}=-8.5 E_{\mathrm{d}}$.

appear in the far field around the zeroth order, that corresponds to the Fourier transform of the Gaussian beam.

To investigate the pattern formation more quantitatively, we define the pattern amplitude $\zeta$ as the ratio of the side-band peaks' power in the optical far field ${ }^{7}$ to the zeroth-order peak:

$$
\zeta=\left\langle\frac{\text { power of the side }- \text { band peaks }}{\text { power of the zeroth order }}\right\rangle_{t} .
$$

Here \langle\rangle$_{\mathrm{t}}$ denotes the temporal average over one oscillation period.

Summarizing our TWM simulations in the broad parameter range of variable saturation $b$, beam ratio $r_{10,2 L}$ and field strength $E_{0}$, there exists a region where periodic spatio-temporal patterns are observed above the instability threshold. Depending on the intensity ratio $r_{10,2 L}$ and the saturation $b$, this region extends over a field strength interval of $\Delta E_{0} \approx 1 E_{\mathrm{d}}$ at low $b$ (by and large independent of $r_{10,2 L}$ ). At higher $b$ these regions are even broader. A change of both the beam ratio in the region of $r_{10,2 L}=25$ to $10^{5}$ and the

\footnotetext{
${ }^{7}$ This corresponds to the sum of the integrals of the squared spectral near field amplitude around $\pm K^{\mathrm{c}}$.
} 

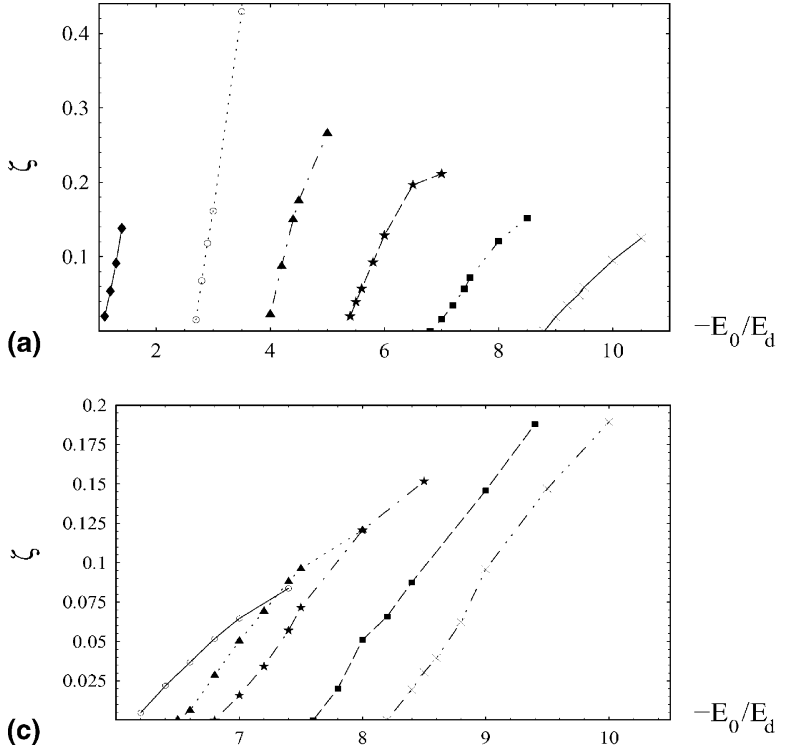
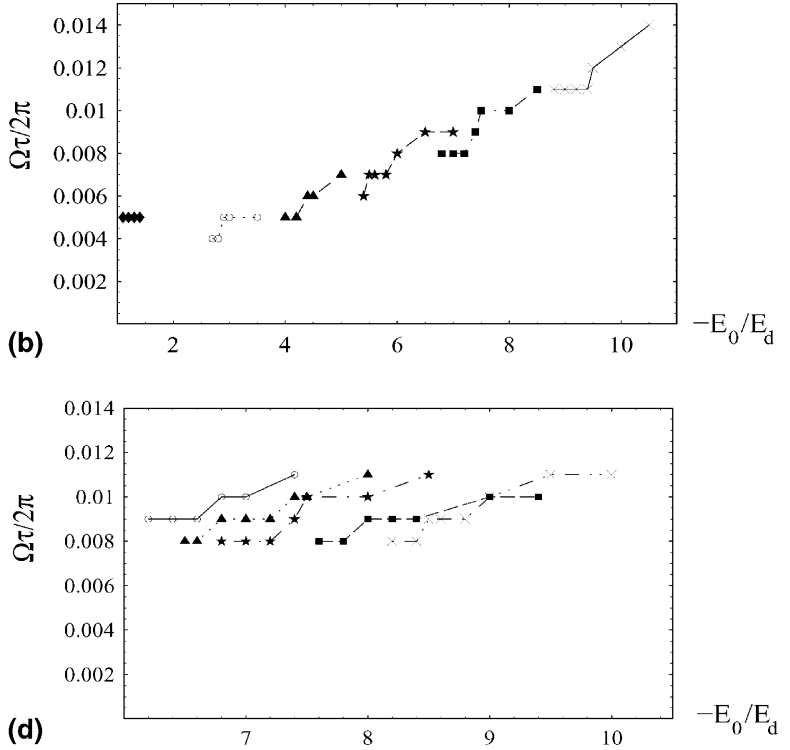

Fig. 8. The pattern amplitude $\zeta$ and the frequency $\Omega$ of the signal beam as a function of the externally applied field strength $E_{0}$ for

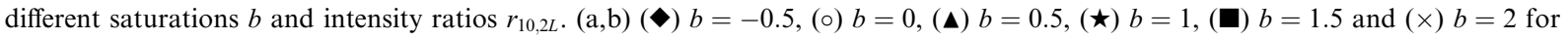
$r_{10,2 L}=250$, respectively, (c,d) (o) $r_{10,2 L}=25,(\boldsymbol{\Delta}) r_{10,2 L}=100,(\star) r_{10,2 L}=250$, (ם) $r_{10,2 L}=1000$ and $(\times) r_{10,2 L}=2500$ for $b=1.5$, respectively.

saturation from $b=-0.5-2$ does not have any qualitative influence on the running transverse waves. The results are shown in Fig. 8, as a function of the bifurcation parameter $E_{0}$.

The pattern amplitude is quickly increasing with $E_{0}$ (starting from $E_{0}^{\mathrm{c}}$ ) at low values of $b$, whereas $\zeta$ grows more slowly when $E_{0}$ is changed at higher $b$ (Fig. 8(a)). As demonstrated by the linear stability analysis (see Fig. 6(a)), the critical field strength $E_{0}^{\mathrm{c}}$ becomes much bigger at higher saturations. For variable intensity ratios $r_{10,2 L}$ the pattern amplitudes possess nearly equal slope and the critical field strengths lie close to each other (Fig. 8(c)). For all parameters supercritical Hopf-bifurcations are observed.

In the same manner the oscillation frequency of the transverse modulation increases above the threshold with the magnitude of $E_{0}$, starting from $\Omega^{\mathrm{c}}$. Thus a higher detuning of the side band compared to the pump beam is predicted. Similar to the pattern amplitudes, the frequencies show nearly the same behaviour for variable beam ratios (Fig. 8(d)), while for various saturations $b$ the oscillation frequencies differ considerably (Fig. 8(b)). This fact can also be observed in Fig. 6(b), where $\Omega^{\mathrm{c}}$ is nearly independent of $r_{10,2 L}$ (for $b=1.5$ ).

\subsection{Signal amplification below and above the instability threshold}

Concerning applications of TWM, the signal beam amplification $g_{\mathrm{S}}$ is one of the interesting physical quantities. It is defined as

$$
g_{\mathrm{S}}:=\left\langle\left(\int_{-\infty}^{\infty} \mathrm{d} x I_{2}(x, z=0)\right) / \int_{-\infty}^{\infty} \mathrm{d} x I_{2}(x, z=L)\right\rangle_{\mathrm{t}}
$$

and is equal to the ratio of the total outgoing to the total incoming energy of the signal beam ${ }^{8}$. Analogously, the gain $g_{\mathrm{P}}$ of the pump beam is defined as

\footnotetext{
${ }^{8}$ The total energy of the beam is obtained by integrating over its transverse beam profile and \langle\rangle$_{\mathrm{t}}$ denotes the temporal average.
} 

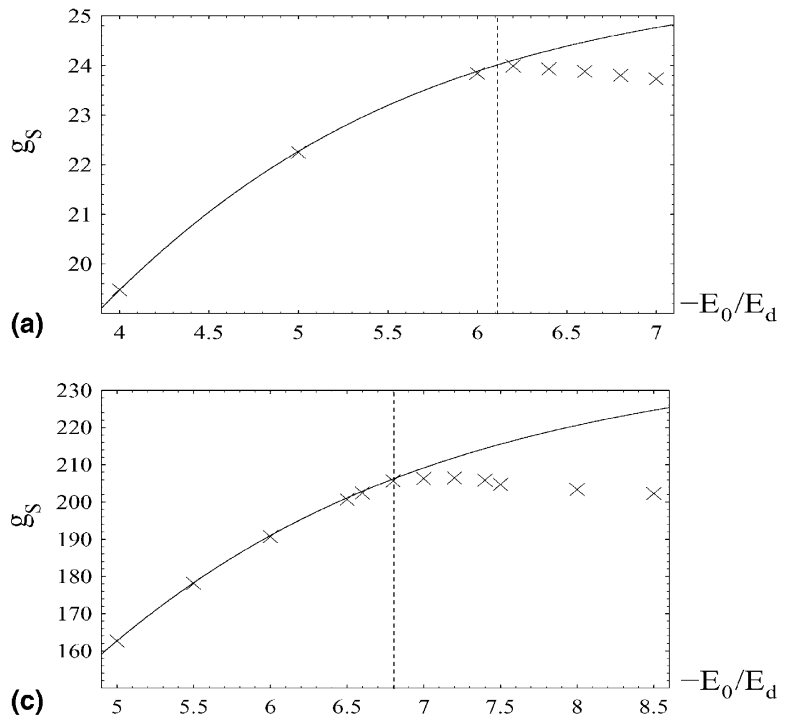
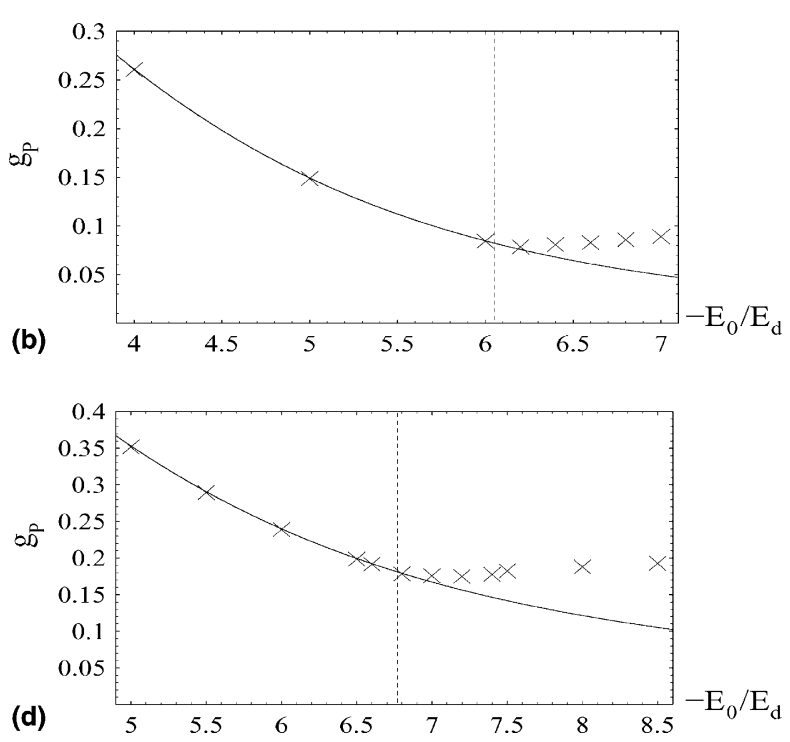

Fig. 9. The gain of the signal and the pump beam $g_{\mathrm{S}}$ and $g_{\mathrm{P}}$ as functions of the field strength for $b=1.5$ and $r_{10,2 L}=25$ (a,b) as well as for $r_{10,2 L}=250(\mathrm{c}, \mathrm{d})$. The numerically determined critical field strength is indicated by a vertical line. $(\times)$ Results for Gaussian beams, (-) results for plane waves using the method of Belić [7].

$$
g_{\mathrm{P}}=\left\langle\left(\int_{-\infty}^{\infty} \mathrm{d} x I_{1}(x, z=L)\right) / \int_{-\infty}^{\infty} \mathrm{d} x I_{1}(x, z=0)\right\rangle_{\mathrm{t}} .
$$

This property measures the energy transfer to the signal beam. Because a modulation depth close to unity has been recognized $(m(z=0) \approx 1)$ as the characteristic of the instability threshold for plane waves, as well as for Gaussian beams (cf. Fig. 4), the critical gain is qualitatively in the order of the intensity ratio $r_{10,2 L}$. In Fig. 9 the gain values of the pump and signal beam, determined by simulations of Gaussian beams, are compared to the ones for stationary plane waves. Below the numerically identified critical field strength, the gain values for Gaussian beams coincide with the calculations for plane waves. Above the threshold, however, a saturation of the gain $g_{\mathrm{S}}$ is observed (Fig. 9(a) and (c)). The results for other parameters are identical. The gain of the pump beam $g_{\mathrm{P}}$ behaves analogously and exhibits a very good agreement between the simulations and semi-analytical values below $E_{0}^{\mathrm{c}}$ and a departure from the plane-wave predictions above the instability threshold (Fig. 9(b) and (d)).

These results are consistent with the requirement of energy conservation. Above the onset of transverse instabilities, the signal beam is no more amplified, as indicated by the saturating gain $g_{\mathrm{S}}$. This energy is now available for the pump beam, and the gain of the pump beam $g_{\mathrm{P}}$ does not decrease any more.

For applications this result implies that, within the confines of the model, signal amplification can only occur up to the critical field strength, beyond which instabilities and pattern formation prevent energy transfer to the signal beam.

\section{Foundations of high-modulation-depth corrections}

In Section 2 pattern formation in a modified TWM model is presented. High-modulation-depth effects are taken into account via a correction function $f(m)$ in Eq. (3), by considering the nonlinear response of the first Fourier component of the space charge field $E_{1}^{\text {sc }}$ with respect to the modulation depth $m$. The correction 
function was chosen to be $f(m)=m /(1+b m)$ [4]. However, at variance with [4], the saturation $b$ has not been restricted to sublinear correction functions $(b>0)$ but has been varied from the superlinear $(b<0)$ to the linear $(b=0)$ and sublinear $(b>0)$ response regime. In this present section of the paper, we attempt to get a better insight into the physical foundations of the correction function $f(m)$. Therefore, we discuss the nonlinear response of the first Fourier component of the space charge field $E_{1}^{\mathrm{sc}}$, based on the underlying KE. For this purpose we decouple the TWM process and assume, that the crystal is exposed to a certain intensity pattern. We determine the response of the crystal. This response shall not influence the intensity pattern in turn. We numerically compute solutions of the KE, placing the emphasis on the buildup of the space charge field. By a Fourier transformation of the electric field in the crystal, we extract the behaviour of the relevant TWM quantity $E_{1}^{\text {sc }}$ (see Eq. (4)), which is responsible for the beam coupling in our model.

In the analysis we will corroborate the expectation of superlinear correction functions in the regime of static gratings, which justifies the consideration of negative saturations $b<0$ in the first part of this paper. The experimentally suggested positive saturations $(b \geqslant 0)$ in the paper by Kwak [4], stated for static gratings, are thus questionable und should probably be negative. Therefore, negative saturations are most relevant in our TWM simulations below the instability threshold, when gratings do not vary with time.

The response of a photorefractive crystal exposed to a laser light is described by Kukhtarev's band transport model [2]

$$
\begin{aligned}
& \partial_{t} N_{\mathrm{D}}^{+}=\left(s I+\beta_{\mathrm{th}}\right)\left(N_{\mathrm{D}}-N_{\mathrm{D}}^{+}\right)-\gamma_{\mathrm{R}} N N_{\mathrm{D}}^{+}, \\
& \partial_{t} N-\partial_{t} N_{\mathrm{D}}^{+}=\frac{1}{e} \vec{\nabla} \cdot \vec{j}, \\
& \vec{j}=\mu\left(e N \vec{E}+k_{\mathrm{B}} T \vec{\nabla} N\right), \\
& \vec{\nabla} \cdot \varepsilon \vec{E}=\rho=e\left(N_{\mathrm{D}}^{+}-N_{\mathrm{A}}^{-}-N\right),
\end{aligned}
$$

where $N$ is the electron density, $N_{\mathrm{A}}^{-}$and $N_{\mathrm{D}}^{+}$are the densities of ionized acceptors and donors, $\vec{j}$ is the current density, $\vec{E}$ is the electric field in the crystal, $\rho$ is the charge density, $I$ is the light intensity, $s$ is the crosssection of photo ionization, $\beta_{\text {th }}$ the thermal excitation rate, $\gamma_{\mathrm{R}}$ the recombination constant, $e=|e|$ the (positive) electron charge, $\mu$ the mobility, $k_{\mathrm{B}}$ Boltzmann's constant, $T$ the temperature, and $\varepsilon$ the dielectric constant. Eqs. (21)-(24) - the KE - are treated numerically, by restricting ourselves to one spatial dimension. This dimension is the longitudinal $z$-direction, because $E_{1}^{\text {sc }}$ is the Fourier component of the space charge field along the grating wave vector $\vec{K}_{\mathrm{g}}$ (cf. Fig. 1). All properties are thus scalar and the spatial gradient is replaced by the derivative $\vec{\nabla} \rightarrow \partial_{z}$.

The crystal is assumed to be exposed to an intensity pattern, which is uniform in $x$ and $y$, with a cosinusoidal profile infinitely extended in the $z$-direction. Under such conditions all other quantities of the crystal are periodic functions, with the period $\Lambda$ of the intensity pattern. To reduce the computational efforts, it is sufficient to solve Eqs. (21)-(24) under periodic boundary conditions on an interval of the length $\Lambda$. This length is approximately $2 \times \lambda=1 \mu \mathrm{m}$ for visible light of wave length $\lambda \approx 500 \mathrm{~nm}$, when the intensity pattern arises from the interference of two counter-propagating beams. However, by tilting the beams, this length $\Lambda$ can be increased, and for the simulations a value of $\Lambda=\lambda / \cos \gamma=10 \mu \mathrm{m}$ has been chosen, based on [20]. In this reference a finitely-extended super-Gaussian intensity distribution, multiplied by a periodic modulation, has been investigated in one dimension, leading to a relatively broad spatial region of periodic response. This justifies our assumptions of periodic boundaries already employed in [21].

Up to now, there have been two ways in the literature of solving Eqs. (21)-(24). In the first method a finite difference scheme is applied, after appropriate scaling [20-23]. Starting from a well-known initial state, the system evolves under the influence of an intensity distribution to a stationary final state. The other method solves a highly nonlinear system of algebraic equations, by utilizing a truncated Fourier series 


$$
g(z)=\sum_{j=-\mathrm{N}}^{\mathrm{N}} \widetilde{g}_{j} \mathrm{e}^{\mathrm{i} j K_{\mathrm{g}} z},
$$

with the grating wave vector $K_{\mathrm{g}}=2 \pi / \Lambda$. This ansatz is substituted into the KE, where $g$ is replaced by $N$, $N_{\mathrm{D}}^{+}, \vec{j}$ and $\vec{E}$. By a minimization process, the Fourier coefficients $\widetilde{g}_{j}$ are determined [8,24]. The disadvantage of this method is the requirement of highly accurate starting values for the Fourier coefficients [22]. The latter procedure suggests, however, to use a spectral method for the solution of the band transport model, rather than the finite differences. By this means, periodic boundary conditions are easily ensured.

The following spectral method represents an alternative method compared to the ones published up to date. After elimination of the current density $\vec{j}$, and by introducing scalings as in [22,23], the discretized equations in $k$-space read

$$
\begin{aligned}
& \partial_{t} \widetilde{v}_{j}=((P-\widehat{P} v)(1+\bar{m} \cos (2 \pi z-\varpi t))-n v)_{j}, \\
& \partial_{t} \widetilde{n}_{j}=\partial_{t} \widetilde{v}_{j}+\frac{K_{\mathrm{g}}^{2}}{K^{2}}\left(\mathrm{i} j \widetilde{n w}_{j}-j^{2} \widetilde{n}_{j}\right), \\
& \widetilde{w}_{j}=\frac{1}{\mathrm{i} j} \frac{\rho_{0}^{2}}{K_{\mathrm{g}}^{2}}\left(\widetilde{v}_{j}-\widetilde{n}_{j}\right) \quad \text { for } j \neq 0, \\
& \widetilde{w}_{0}=\frac{\widetilde{e E_{0}}}{K_{\mathrm{g}} k_{\mathrm{B}} T} .
\end{aligned}
$$

The new variables are ${ }^{9}$ : the electron density $n=N / N_{\mathrm{A}}$, the ionized donor density $v=N_{\mathrm{D}}^{+} / N_{\mathrm{A}}$ and the space charge field $w=e E /\left(k_{\mathrm{B}} T K_{\mathrm{g}}\right)=E / E_{\mathrm{d}}$ in scaled form, respectively. The effective photoionization rate $G_{0}=s I_{0}+\beta_{\text {th }}$ enters the quantities $P=G_{0} N_{\mathrm{D}} /\left(\gamma_{\mathrm{R}} N_{\mathrm{A}}^{2}\right), \widehat{P}=G_{0} /\left(\gamma_{\mathrm{R}} N_{\mathrm{A}}\right)$, furthermore $K^{-2}=\mu k_{\mathrm{B}} T /\left(e \gamma_{\mathrm{R}} N_{\mathrm{A}}\right)$, $\rho_{0}^{2}=e^{2} N_{\mathrm{A}} / \varepsilon k_{\mathrm{B}} T$, the scaled frequency detuning $\varpi=\delta \omega /\left(\gamma_{\mathrm{R}} N_{\mathrm{A}}\right)$ and the effective modulation depth $\bar{m}=\left(1+\beta_{\text {th }} /\left(s I_{0}\right)\right)^{-1} m$ have been introduced. Each $z$-dependent quantity has been expanded as in Eq. (25), where tildes denote Fourier components, and $j$ is an integer. The variable $t$ is the time $t / \tau_{\mathrm{R}}=t \gamma_{\mathrm{R}} N_{\mathrm{A}}$, scaled with the recombination time constant $\tau_{\mathrm{R}}=\left(\gamma_{\mathrm{R}} N_{\mathrm{A}}\right)^{-1}$, and $z$ represents the scaled longitudinal direction $z / \Lambda$. Compared to the scalings applied for the TWM equations, it is obvious that these equations describe the microscopic regime. The crystal length $L$ is about three orders of magnitude bigger then $\Lambda$, and the photorefractive time constant $\tau=N_{\mathrm{A}} /\left(N_{\mathrm{D}} S I_{0}\right) \approx 9 \times 10^{-3} \mathrm{~s}$ is about three orders of magnitude larger than $\tau_{\mathrm{R}} \approx 6 \times 10^{-6} \mathrm{~s}$ for the parameters used here.

To complete the system of Eqs. (26)-(29), the charge conservation is added for each wave length interval

$$
\int_{0}^{1} \frac{\rho}{N_{\mathrm{A}}} \mathrm{d} z=\int_{0}^{1}(v-1-n) \mathrm{d} z=0,
$$

which in Fourier space is written as a condition

$$
\widetilde{n}_{0}=\widetilde{v}_{0}-\widetilde{1} \text {. }
$$

The integration scheme is the following: (i) temporal integration of the first two equations (26) and (27), using an explicit Runge-Kutta-scheme of fourth order, (ii) determination of $\widetilde{w}$, according to Eq. (28), after each time step, (iii) control of the zeroth order of $\widetilde{w}$ and $\widetilde{n}$, because of the externally applied field (Eq. (29)) and the space charge conservation (Eq. (31)), (iv) fulfillment of the condition of periodicity and reality of

\footnotetext{
${ }^{9}$ As the acceptors are assumed to be totally ionized, $N_{\mathrm{A}}^{-}$is replaced by $N_{\mathrm{A}}$ in the following.
} 
functions in the real space, by demanding that $\widetilde{n}_{j}=\widetilde{n}_{-j}^{*}, \widetilde{v}_{j}=\widetilde{v}_{-j}^{*}$ and $\widetilde{w}_{j}=\widetilde{w}_{-j}^{*}$. Because of condition (iv), only one side of the spectrum needs to be computed.

The initial values are chosen such that the light is switched on at $t=0$. For $t<0$ the intensity is therefore $I=0$, and the crystal is in a stationary, spatially homogeneous state $\left(\partial_{t}=0\right.$ and $\left.\partial_{x}=0 \cong k=0\right)$ :

$$
\begin{aligned}
& n(z)=\frac{1}{2}\left(-1+\sqrt{4 P+(1-\widehat{P})^{2}}-\widehat{P}\right), \\
& v(z)=\frac{1}{2}\left(1+\sqrt{4 P+(1-\widehat{P})^{2}}-\widehat{P}\right), \\
& w(z)=\frac{e E_{0}}{k_{\mathrm{B}} T K_{\mathrm{g}}} .
\end{aligned}
$$

Neglecting thermal effects, $n$ is 0 at the starting time because there exist no excited electrons. $v$ in contrary is equal to 1 under these conditions (Eq. (31)) and $w$ is given by the external electric field. For $t>0$ a light field is present in the crystal, with the following intensity distribution:

$$
I(z, t)=I_{0}(1+m \cos (2 \pi z-\varpi t)) .
$$

In the case of $\varpi=0$, Eq. (35) represents static gratings, otherwise running gratings. The crystal parameters are chosen according to Ref. [20] and are based on experimental data, describing a $\mathrm{Bi}_{12} \mathrm{SiO}_{20}$-crystal (BSO): $N_{\mathrm{D}}=10^{25} \mathrm{~m}^{-3}, \quad N_{\mathrm{A}}=0.95 \times 10^{22} \mathrm{~m}^{-3}, \mu=10^{-5} \mathrm{~m}^{2} / \mathrm{V} \mathrm{s}, \gamma_{\mathrm{R}}=1.65 \times 10^{-17} \mathrm{~m}^{3} / \mathrm{s}, \quad s=1.06 \times 10^{-5} \mathrm{~m}^{2} / \mathrm{J}$ and $\varepsilon=9.7 \varepsilon_{0}$.

The length of the intensity period was set to $\Lambda=10 \mu \mathrm{m}$ (as mentioned), and the intensity of the incident laser radiation to $I_{0}=10^{4} \mathrm{~W} / \mathrm{m}^{2}$. The influence of the thermal excitation $\beta_{\text {th }}$ in Eqs. (26)-(29) is only contained in $\bar{m}$. Therefore, the effective intensity contrast in the photorefractive crystal is reduced by thermal effects. To investigate the influence of high modulation depth, we assume a negligible thermal excitation rate $\left(\beta_{\text {th }}=0\right)$. In the following, we will investigate both stationary $(\varpi=0)$ and running intensity distributions $(\varpi \neq 0)$ at room temperature $(T=293 \mathrm{~K})$. The applied field $E_{0}$ is varied between 0 and $5 \times 10^{4} \mathrm{~V} / \mathrm{m}$, and the modulation depth of the intensity profiles, present in the crystal, is altered from $m=0$ to $m=1$ (Fig. 10).

We remark that the parameters of the BSO-crystal used in this part differ from the $\mathrm{BaTiO}_{3}$-crystal discussed in TWM part. This is for reasons of comparison, because up to now most of the simulations on running gratings have been performed for BSO crystals [8,21]. The internal fields of the BSO-crystal are: $E_{\mathrm{d}}=k_{\mathrm{B}} T K_{\mathrm{g}} / e=1.59 \times 10^{4} \mathrm{~V} / \mathrm{m}, \quad E_{\mathrm{q}}=e N_{\mathrm{A}} /\left(\epsilon K_{\mathrm{g}}\right)=2.82 \times 10^{7} \mathrm{~V} / \mathrm{m} \approx 1.77 \times 10^{3} E_{\mathrm{d}} \quad$ and $\quad E_{\mu}=\gamma_{\mathrm{R}} N_{\mathrm{A}} /$ $\left(\mu K_{\mathrm{g}}\right)=2.49 \times 10^{4} \mathrm{~V} / \mathrm{m} \approx 1.57 E_{\mathrm{d}}$.

In simulations a grid of 512 points in the longitudinal direction of the crystal is used. A time step of $\tau_{\mathrm{R}}=\left(\gamma_{\mathrm{R}} N_{\mathrm{A}}\right)^{-1}$ needs to be divided at least into 5000 smaller steps, to obtain numerical stability for the integration procedure. Furthermore, we are using a Fermi-filter, which provides an increased damping for modes, starting from the 100th harmonic and up to $k_{\max }=512 / 2$. This additional damping is justified by the results presented in [24], where only Fourier coefficients up to $\mathrm{N}=50$ have been used in the algebraic solutions of the KE.

\subsection{The nonlinear response of the photorefractive crystal}

In this section numerically obtained solutions for the space charge field are presented, considering the nonlinear response of the medium. We display the stationary and steady-state space charge field for static 


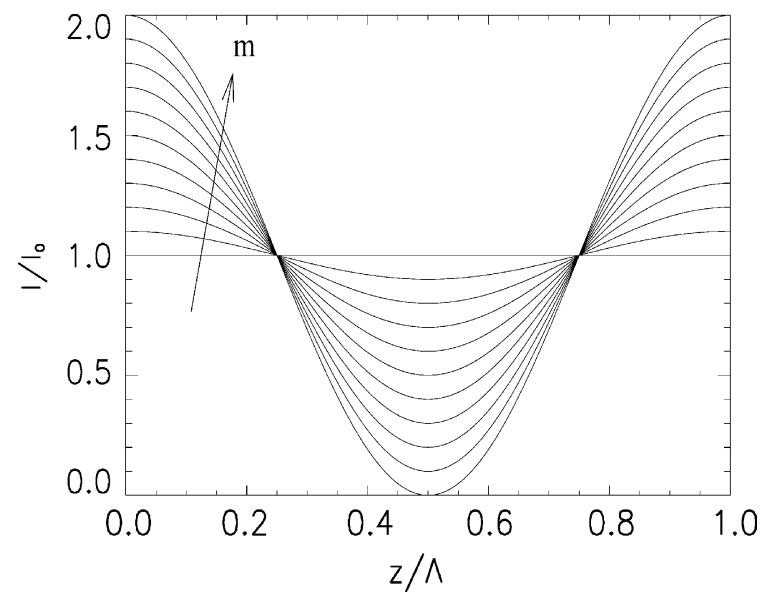

Fig. 10. Stationary intensity distributions present in the crystal for $m=0,0.1, \ldots, 1$. The modulation depth is increasing in the direction indicated by the arrow.

and running intensity distributions, respectively. To compute such solutions, the integration time is chosen long enough to eliminate transients.

\subsubsection{Static intensity distributions}

In Fig. 11 the electric field in the crystal is shown for the stationary intensity distributions given in Fig. 10 , under the influence of different externally applied fields $E_{0}$. Results for the maximum modulation depth $m=1$ are not depicted, because the convergence is slow and transients persist very long. To reach a stationary state, simulations have been performed for time intervals between $\Delta t \approx 10^{3} \tau_{\mathrm{R}}$ for small $m$, up to $\Delta t \approx 10^{4} \tau_{\mathrm{R}}$ for $m=0.9^{10}$. According to the KE, the field strength $E$ measures the total electric field in the crystal. Owing to the externally applied field $E_{0}$, the total electric field is $E=E^{\text {sc }}+E_{0}$, where $E^{\text {sc }}$ solely contains the response of the crystal.

For small modulation depth ( $m=0.1$ and 0.2$)$, and without an externally applied field (Fig. 11(a)), a sinusoidal electric field is obtained as a response to the cosinusoidal intensity distribution. These solutions correspond to the approximate small- $m$ solutions given in [1,2], displaying a phase shift of $\pi / 2$ with respect to the intensity pattern. For higher modulation depth a strongly nonlinear response is observed. The gradients at $z=0.5$ become gradually steeper, preventing the diffusion of charge carriers into the dark region. The sign of the space charge field switches at the minimum of the intensity $(z=0.5)$, to oppose the diffusion by its drift forces. The first Fourier component of the space charge field is purely imaginary, owing to the point symmetry around $z=0$.

In Fig. 11(b) and (c) an external field, pointing into the positive $z$-direction, is applied to the crystal. It can clearly be seen how the external field destroys the point symmetry around $z=0.5$. In Fig. 11(b) a phase shift of the space charge field develops, compared to Fig. 11(a), as well as an asymmetry between the maximum and minimum value of $E$, which is not simply due to the bias $E_{0}=0.31 E_{\mathrm{d}}$. Because of the phase shift, the first Fourier component of the space charge field is no more purely imaginary. This effect is contained in the $E_{0}$-dependent coefficients $\eta$ and $\Gamma$ in Eq. (3). The higher field strength $E_{0}=3.1 E_{\mathrm{d}}$ (Fig. 11(c)) has brought us to the drift-dominated regime in contrary to the diffusion-dominated regime in Fig. 11(a). In Fig. 11(c) the electric field resembles a negative cosine curve for small modulation depths, similar

\footnotetext{
${ }^{10}$ The time intervals given correspond to $E_{0}=3.1 E_{\mathrm{d}}$. They depend on the applied field and can be reduced for a lower $E_{0}$.
} 


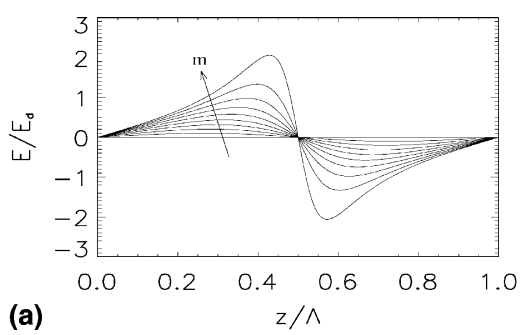

(a)
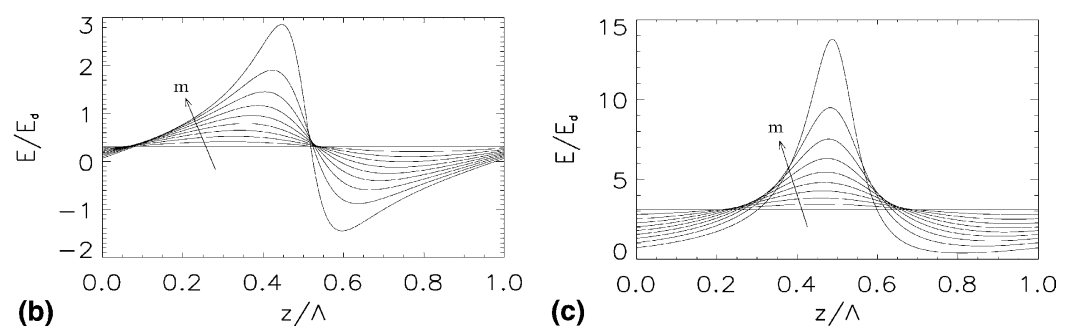

Fig. 11. The total electric field in the crystal $E=E_{0}+E^{\text {sc }}$ for different applied external fields: (a) $E_{0}=0$, (b) $E_{0}=0.31 E_{\mathrm{d}}$, (c) $E_{0}=3.1 E_{\mathrm{d}}$. The modulation depth is varied from $m=0$ to $m=0.9$ in steps of $\Delta m=0.1$. The direction of increasing $m$ is indicated by the arrow.

to the analytical approximate solutions presented in [25] for the case without diffusion. The phase shift between the interference pattern and the space charge field is almost $\pi$. As already observed in Fig. 11(a) and (b), the spectra of $E$ reveal high amplitudes of the higher harmonic terms, especially for $m=0.9$. The trends visible in Fig. 11(b) are much more pronounced in Fig. 11(c). The maximum of the space charge field has strongly grown for high $m$ and is shifted to the right, which is due to the higher field $E_{0}$, pulling the electrons to the right of the symmetry point $z=0.5$. The minimum in the field strength to the right of $z=0.5$ is washed out, and the asymmetry between values below the bias field $E_{0}$ and above it is apparent. As these effects are observable, especially for a high modulation depth, they are evidently caused by the nonlinear character of the KE.

Other internal crystal quantities, as for example the electron density and the ionized donor density, have not been represented here. Interestingly - as reported in $[23,26]$ - a nearly perfect cosinusoidal response of the excited electron density is observed for different modulation depths $m$ and even for the different externally applied fields $E_{0}$. This, in a way, runs against the first intuition, that the applied $E_{0}$ should break the symmetry around $z=0.5$ in all crystal's internal variables. However, it turns out that the excited electron density simply "copies" the intensity distribution, and the second harmonics are already orders of magnitude smaller than the first Fourier components of $n\left(\widetilde{n}_{2} \ll \widetilde{n}_{1}\right)$.

\subsubsection{Running intensity distributions}

We now investigate the response of the crystal to running intensity distributions, given by Eq. (35) with $\varpi \neq 0$. The term $\exp \left[\mathrm{i}\left(\psi_{1}-\psi_{2}\right)\right]$ in Eq. (3) is replaced by $\exp (-\mathrm{i} \delta \omega t)$, which acts as an oscillatory forcing term. This time dependence arises, because the phase difference $\psi_{1}-\psi_{2}$ in Eq. (3) is equal to $-\delta \omega t$, owing to the special form of the intensity pattern in Eq. (35). If the imaginary part of $\eta$ is at the same frequency as the forcing term, a resonance phenomenon can be expected (provided the real part of $\eta$ is small), leading to a high first-order harmonic that should strengthen the interaction between the counter-propagating beams in a TWM experiment. Carrying out the calculations as described in [3], and scaling with the recombination time $\tau_{\mathrm{R}}=\left(\gamma_{\mathrm{R}} N_{\mathrm{A}}\right)^{-1}$ yields an optimum value of the frequency detuning

$$
\varpi_{\mathrm{opt}} \approx 0.24 \text {. }
$$

Another possibility to compute the frequency at which the first Fourier component takes its maximum value, is the decomposition of all crystal's internal quantities in terms of $\exp ( \pm \mathrm{i}(2 \pi z-\varpi t))$. Using Eq. (21) from [3] one obtains

$$
\varpi_{\mathrm{opt}} \approx 0.28 \text {. }
$$

Henceforth simulations with running gratings are carried out with $\varpi=0.25$. A high amplitude of the first Fourier component of the induced space charge field is expected for this frequency (Fig. 12). The space 


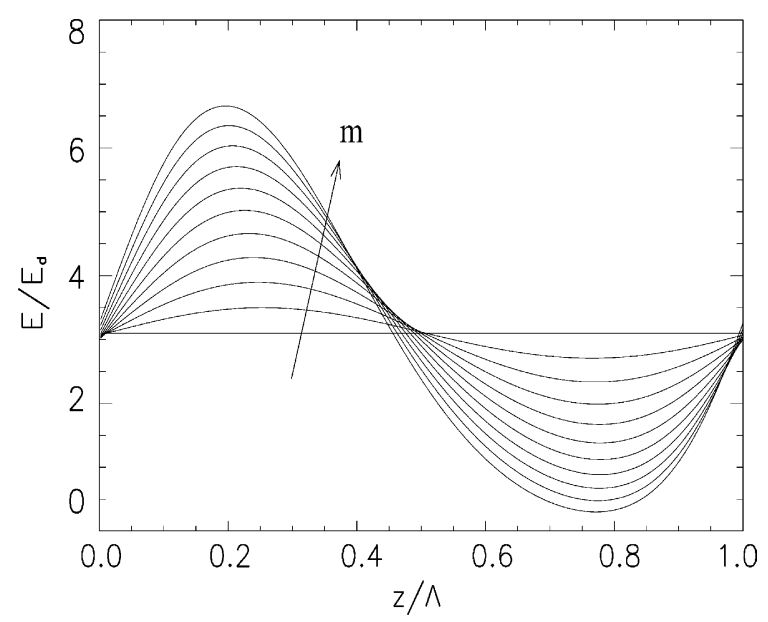

Fig. 12. The total electric field in the crystal $E=E_{0}+E^{\mathrm{sc}}$ for an applied external field of $E_{0}=3.1 E_{\mathrm{d}}$ and an intensity pattern running with a frequency of $\varpi=0.25$. The modulation depth is varied from $m=0$ to $m=1.0$ in steps of $\Delta m=0.1$. The direction of increasing $m$ is indicated by the arrow and the picture is taken at $t \approx 502.7 \tau_{\mathrm{R}}$, corresponding to 20 temporal periods.

charge field resembles a sine-function for small $m$, similar to the diffusion-dominated regime (cf. Fig. 11(a)). But there is an increasing asymmetry around $z=0.5$ for higher modulation depths. A modulation-depthdependent-phase delay, as compared to the cosinusoidal intensity distribution, can be observed, namely the intersections with the bias electrical field shifted to the left for high modulation depth $m$. This delay gives rise to a modulation-depth-dependent phase of the correction function $f(m)$, as will be discussed in Section 3.2. Furthermore, the situation in Fig. 12 can be compared to the results shown in Fig. 11(c), because the applied field strength $E_{0}$ is the same and the intensity pattern is taken again in the starting position (cf. Fig. 10). Obviously, the movement of the fringes leads to a totally different response of the crystal. Besides the sinusoidal instead of the cosinusoidal shape of the electric field $E$, the maximum and the minimum of the electric field approximately exhibit the same distance from the bias voltage, another considerabe difference to Fig. 11(c).

\subsection{Physically motivated response functions}

Starting from the space charge field solutions presented in Section 3.1, we want to get a better insight into the nonlinear behaviour of the first Fourier component. To recall, this component is responsible for the beam coupling in our TWM model and its nonlinear modulation-depth dependence has been taken into account via the function $f(m)$ in Eq. (3). To determine a response function based on the underlying KE, we solve Eq. (3) for the stationary state, when all transients have died away. This solution reads

$$
Q=\frac{\Gamma}{2(\eta-\mathrm{i} \delta \omega \tau)} f(m) \mathrm{e}^{\mathrm{i}\left(\psi_{1}-\psi_{2}\right)},
$$

where the phase difference $\psi_{1}-\psi_{2}$ is equal to $-\delta \omega t$ (see Section 3.1.2) and an ansatz $Q(t)=Q_{0} \exp [\mathrm{i}(-\delta \omega t)]$ has been used in Eq. (3). The frequency $\delta \omega$ is equal to 0 for static interference patterns, and $\delta \omega \neq 0$ corresponds to moving interference patterns.

Before determining the correction function, we interpret the physical meaning of Eq. (38) with respect to the results of the simulations on static and running gratings in Section 3.1. The phase of $Q$ is linearly influenced by the phase of the interference pattern $\psi_{1}-\psi_{2}$ due to the term $\exp \left(\mathrm{i}\left(\psi_{1}-\psi_{2}\right)\right)$. This linear phase 
response is consistent with the model of an infinitely extended crystal, which is exposed to a periodic intensity pattern. Evidently, in this situation a phase shift of the interference pattern causes a phase shift of the space charge field by the same amount, owing to the periodic boundary conditions. Analogously, a complex correction function $f(m)$, instead of a real function, physically corresponds to a modulationdepth-dependent-phase shift of the space charge field.

The first Fourier component of the (scaled) space charge field $\widetilde{w}_{1}$ is, according to Eq. (38), proportional to $f(m)$ via the connection $\widetilde{w}_{1} \propto E_{1}^{\text {sc }} \propto Q$ (cf. Eq. (4)). Thus, the high- $m$-correction function can be determined via a simple scaling law

$$
f(m)=\frac{\widetilde{w}_{1}(m)}{\widetilde{w}_{1}\left(m_{\mathrm{s}}\right)} m_{\mathrm{s}} .
$$

Here $m_{\mathrm{s}}$ is a sufficiently small modulation depth, for which the analytical solution in the frame of the smallmodulation-depth approximation holds. (Remember $f(m)=m$ for $m \ll 1$.) Therefore, $f(m)$ is computed by relating the first-order Fourier components of the space charge field for equal $E_{0}$, the same frequency $\varpi=\delta \omega /\left(\gamma_{\mathrm{R}} N_{\mathrm{A}}\right)$ and identical phase difference of the interference pattern $\left(\psi_{1}-\psi_{2}\right)$. The requirement for the identical phase difference implies, that for running gratings the space charge field components $\widetilde{w}_{1}$ for the same times have to be used in Eq. (39). For extracting $f(m)$, we use $m_{\mathrm{s}}=0.01$ for the small modulation depth. To be sure that the first Fourier component of the space charge field has converged into a stationary state (which is described by Eq. (38)), we multiply this quantity with a factor of $\exp (\mathrm{i} \varpi t)$, to get rid of its fast time dependence (time scaled to $\tau_{\mathrm{R}}$ ). A temporally constant value of $\widetilde{w}_{1} \exp (\mathrm{i} \varpi t)$ indicates convergence.

The plot of the real part of $f(m)$ as a function of the modulation depth $m$, according to Eq. (39), is shown in Fig. 13 for static and running gratings. Additionally the (real) linear response $f(m)=m$ has been plotted, together with a (real) superlinear and a (real) sublinear response $f(m)=m /(1+b m)$ with $b=-0.5$ and $b=1.5$, respectively. The curves for static intensity distributions are all the same, independent of the applied field strength $E_{0}$. The real part of the correction function behaves superlinearly for static intensity patterns. It can quite well be fitted with a $b \approx-0.3$, even if the real part has some other functional dependence for high $m$ than reproducable by $m /(1+b m)$. However, the real part of $f(m)$ exhibits a sublinear response for a running grating $(\varpi=0.25$ in this case). A saturation $b \approx 0.25$ would fit the curve $\operatorname{Re}(f(m))$.

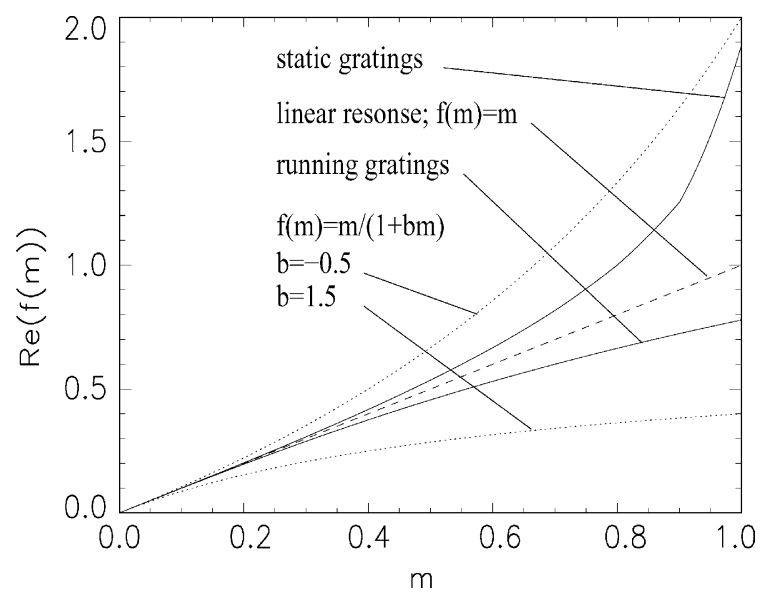

Fig. 13. The real part of the physically motivated correction functions $f(m)$ as determined from exact simulations of the Kukhtarev equations. The response for the static and dynamic cases is indicated. Additionally the linear response $f(m)=m$ and $f(m)=m /(1+b m)[4]$ are displayed. 


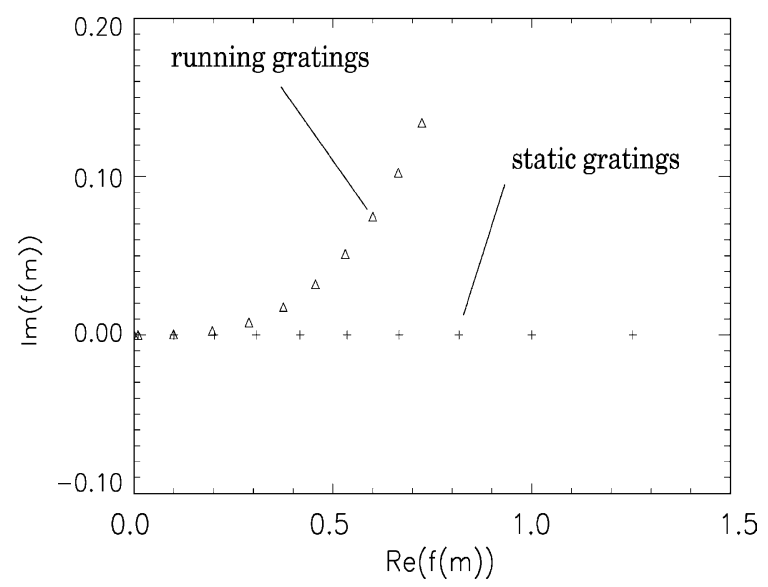

Fig. 14. The real and imaginary parts of the computed modulation-depth-dependent-correction function $f(m)$ for $(+)$ static and $(\Delta)$ running gratings. The modulation depth has been varied from $m=0$ to $m=0.9$ in steps of $\Delta m=0.1$.

This behaviour of the real part of $f(m)$ is analogous to the results in Refs. [8,9], where it was demonstrated that for running intensity patterns, based on the KE, a sublinear response is obtained.

Owing to the complex coefficients $\widetilde{w}_{1}$ in Eq. (39), the modulation-depth-correction function $f(m)$ should generally be considered complex (Fig. 14). Even in the complex plane the computed curves for static intensity distributions are identical, independent of the applied field strengths $E_{0}$. There is no numerically relevant imaginary component observed, so that the correction function is completely real $(f(m)=\operatorname{Re}(f(m)))$. Hence, the nonlinear response of the crystal is, in the static regime, fully described by a superlinear real correction function. This is in agreement with results given in [21,24,27] (for different crystal parameters), where the statement was made, that the amplitude of the first Fourier component is larger than predicted by the small- $m$ approximation. However, for running ratings a pronounced imaginary part occurs. This fact is due to the modulation-depth-dependent-phase shift shown in Fig. 12. To our knowledge, this effect has been reported here for the first time. The majority of papers published on TWM only discuss effects related to intensities, such as gain, etc. Phase effects in TWM are mostly ignored, and with them also the modulation-depth-dependent-phase shift for dynamic gratings.

For running gratings a complex correction function of the form

$$
f(m)=|f(m)| \mathrm{e}^{\mathrm{i} \varphi(m)}
$$

is required for a more realistic description of a photorefractive crystal. The exact shape of $f(m)$ can be determined by making use of the method explained in this paper. Providing the relevant experimental quantities (the frequency of the running intensity pattern and the specific parameters of the crystal), the procedure involves mainly two steps: (i) Solve the KE and extract the first Fourier component of the space charge field and (ii) compute $f(m)$ according to Eq. (39).

\section{Conclusions}

In the present contribution the influence of a high modulation depth on pattern formation and the foundation of the high-modulation-depth correction are discussed. For this purpose we start from a simple model of contra-directional TWM in photorefractive crystals, and modify it by a correction function, to account for the experimentally observed modulation-depth effects. 
A linear stability analysis is carried out on this model. By integration of the stability matrix - extended beyond the special case of the "modulation depth one" - the non-autonomous problem is solved for a modulation depth, which changes along the direction of the beam propagation. An instability threshold is found for different beam ratios $r_{10,2 L}$, applied field strengths $E_{0}$ and saturation parameters $b$, ranging from a sublinear to a superlinear response. The results from numerical simulations of TWM for Gaussian beams are in very good agreement with the critical quantities predicted by the linear stability analysis.

The influence of a high saturation on the pattern formation shows up in an increase of the critical electric field strength and generally a stronger critical detuning of the side-band beams. As a characteristic feature of pattern formation, a modulation depth close to unity is required at that side of the crystal, where the pump beam enters. Even for small signal and high pump beam intensities, pattern formation arises, if the external field strength is adjusted in such a way that a modulation depth of approximately unity is achieved. Therefore, effects of a high modulation depth have to be considered when pattern formation in the contradirectional configuration is discussed.

Compared to the case of linear response [17], besides transverse running waves, no qualitatively new patterns are observed near and above the instability threshold. Independent of the saturation, the simulations show periodic, symmetric beam profiles. Quantitatively, however, the TWM simulations reveal that a stronger sublinear response of the crystal leads in general to a stronger detuning of the side-band beams, and the pattern amplitude increases more slowly with respect to the applied field strength.

The simulations of the TWM equations show that the gain, i.e. the amplification of the signal beam, saturates above the instability threshold. Pattern formation therefore prevents a further enhancement of the signal.

In the second part of the paper, we investigate the physical foundations of the high-modulation-depth corrections. Trying to justify the presumed form of the correction functions, used in $[3,4]$ to explain experimental results, we develop a new spectral integration scheme, which exactly solves the KE.

The response of the space charge field to static and running intensity distributions is explored for different applied external electric fields. Phase shifts due to the external field are demonstrated, together with the transition from the diffusion-dominated crystal response and the drift-dominated regime. These observations motivate a superlinear response of the investigated BSO-crystal for stationary intensity distributions. Recently the superlinear behaviour has been measured in experiments on TWM in a $\mathrm{KNbO}_{3}$ crystal [28]. This substantiates that the sublinear correction function proposed by Kwak [4] for static gratings is not appropriate, but one should utilize negative saturations in this regime. For running gratings complex correction functions (with a sublinear real part) are necessary, to take properly the modulationdepth effects into account.

Starting from the results of the second part of the paper, it is now desirable to develop a consistent model of photorefractive TWM. For a high modulation depth the amplitudes of the higher harmonics of the space charge field become comparable to the amplitude of the first-order component. An investigation of how strongly these higher harmonics, so far neglected owing to a resonance argument, influence the TWM process, could present an interesting topic. It might well be, that the experimentally suggested correction functions not only describe the nonlinear behaviour of the first-order harmonic of the space charge field, but also "purely phenomenological" modifications due to the coupling of "higher harmonic gratings".

If it turns out that, indeed, the first-order harmonic of the space charge field is the most relevant for the beam interactions, the complex correction function needs to be consistently included in the TWM model. Especially for the dynamic transverse instabilities a proper consideration of the transition from a superlinear real correction function to a complex correction function has to be performed, because the static gratings change to running gratings above the instability threshold. For this purpose one should use the explored negative values of the saturation $b$ in our TWM model below the threshold, corresponding to a superlinear response. As soon as temporal patterns or transient effects appear one should adapt the correction function continuously to become a complex valued function. A fully consistent model, where the 
solutions of the $\mathrm{KE}$ are coupled to the propagation equations in three spatial dimensions, represents a challenging project for future work.

\section{References}

[1] N.V. Kukhtarev, V.B. Markov, S.G. Odulov, M.S. Soskin, V.L. Vinetskii, Ferroelectrics 22 (1979) 949.

[2] P. Yeh, Introduction to Photorefractive Nonlinear Optics, Wiley, New York, 1993.

[3] Ph. Refregier, L. Solymar, H. Rajbenbach, J.P. Huignard, J. Appl. Phys. 58 (1985) 45.

[4] C.H. Kwak, S.Y. Park, J.S. Jeong, H.H. Suh, E. Lee, Opt. Commun. 105 (1994) 353.

[5] V. Dupray, M. Brunel, C. Özkul, N. Wolffer, J. Opt. Soc. Am. B 17 (2000) 407.

[6] J.E. Millerd, E.M. Garmire, M.B. Klein, B.A. Wechsler, F.P. Strohkendl, J. Opt. Soc. Am. B 9 (1992) 1449.

[7] M.R. Belić, D. Timotijević, M. Petrović, M.V. Jarić, Opt. Commun. 123 (1996) 201.

[8] L.B. Au, L. Solymar, Opt. Lett. 13 (1988) 660.

[9] G.A. Brost, K.M. Magde, J.J. Larkin, M.T. Harris, J. Opt. Soc. Am. B 11 (1994) 1764.

[10] T. Honda, Opt. Lett. 18 (1993) 598.

[11] T. Honda, Opt. Lett. 20 (1995) 851.

[12] P.P. Banerjee, H.-L. Yu, D.A. Gregory, N. Kukhtarev, H.J. Claufeld, Opt. Lett. 20 (1995) 10.

[13] M. Schwab, M. Sedlatschek, B. Thüring, C. Denz, T. Tschudi, Chaos Solitons Fractals 10 (1998) 701.

[14] C. Denz, M. Schwab, M. Sedlatschek, T. Tschudi, T. Honda, J. Opt. Soc. Am. B 15 (1998) 2057.

[15] M. Saffman, D. Montgomery, A.A. Zozulya, D.Z. Anderson, J. Opt. Soc. Am. B 11 (1994) 1409.

[16] T. Honda, P.P. Banerjee, Opt. Lett. 21 (1996) 779.

[17] O. Sandfuchs, F. Kaiser, M.R. Belić, J. Opt. Soc. Am. B 15 (1998) 2070.

[18] O. Sandfuchs, F. Kaiser, M.R. Belić, Phys. Rev. A 64 (2001) 063809.

[19] W. Krolikowski, M.R. Belić, M. Cronin-Golomb, A. Bledowski, J. Opt. Soc. Am. B 7 (1990) 1204.

[20] N. Singh, S.P. Nadar, P.P. Banerjee, Opt. Commun. 136 (1994) 487.

[21] G.A. Brost, J. Opt. Soc. Am. B 9 (1992) 1454.

[22] Y.H. Lee, R.W. Hellwarth, J. Appl. Phys. 71 (1992) 916.

[23] P. Buchhave, J. Opt. Soc. Am. B 15 (1998) 1865.

[24] E. Serrano, V. López, M. Carrascosa, F. Agulló-López, IEEE J. Quantum Electron. 30 (1994) 875.

[25] F. Vachs, L. Hesselink, J. Opt. Soc. Am. A 5 (1988) 690.

[26] N.V. Kukhtarev, P. Buchhave, S.F. Lyuksyutov, Phys. Rev. A 55 (1997) 3133.

[27] A. Bledowski, J. Otten, K.H. Ringhofer, Opt. Lett. 16 (1991) 672.

[28] D. Tamer, Diploma Thesis, Darmstadt University of Technology, 2000. 